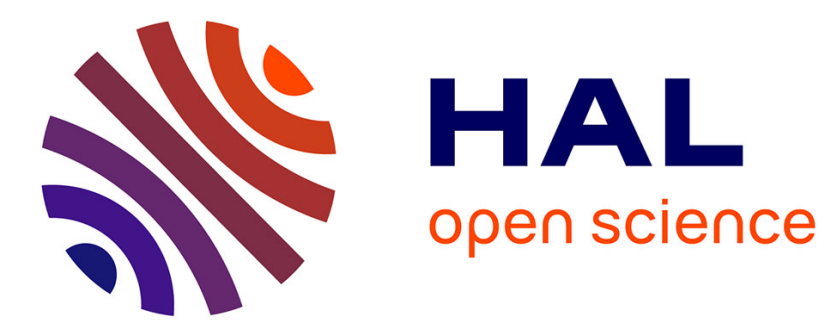

\title{
Quaternion correlation for tracking crystal motions
}

\author{
Qiwei Shi, Félix Latourte, François Hild, Stéphane Roux
}

\section{To cite this version:}

Qiwei Shi, Félix Latourte, François Hild, Stéphane Roux. Quaternion correlation for tracking crystal motions. Measurement Science and Technology, 2016, 27, pp.95006 - 95006. 10.1088/09570233/27/9/095006 . hal-01383950

\section{HAL Id: hal-01383950 https://hal.science/hal-01383950}

Submitted on 19 Oct 2016

HAL is a multi-disciplinary open access archive for the deposit and dissemination of scientific research documents, whether they are published or not. The documents may come from teaching and research institutions in France or abroad, or from public or private research centers.
L'archive ouverte pluridisciplinaire $\mathbf{H A L}$, est destinée au dépôt et à la diffusion de documents scientifiques de niveau recherche, publiés ou non, émanant des établissements d'enseignement et de recherche français ou étrangers, des laboratoires publics ou privés. 


\title{
Quaternion correlation for tracking crystal motions
}

\author{
Qiwei Shi ${ }^{1,2}$, Félix Latourte ${ }^{2}$, François Hild ${ }^{1}$, Stéphane Roux ${ }^{1}$ \\ 1: LMT-Cachan, ENS Cachan / CNRS / Université Paris-Saclay, \\ 61 avenue du Président Wilson, F-94235 Cachan (FRANCE) \\ 2: EDF R\&D, Site des Renardières, \\ avenue des Renardières, Ecuelles, F-77818 Moret-sur-Loing (FRANCE) \\ Received: date / Accepted: date
}

\begin{abstract}
During in-situ mechanical tests performed on polycrystalline materials in a scanning electron microscope, crystal orientation maps may be recorded at different stages of deformation from electron backscattered diffraction (EBSD). The present study introduces a novel correlation technique that exploits the crystallographic orientation field as a surface pattern to measure crystal motions. Introducing a quaternion-based formalism reveals very convenient to handle crystal symmetry and orientation extraction. Spatial regularization is provided by a penalty to deviation of displacement fields from being the solution to a homogeneous linear elastic problem. This procedure allows the large scale features of the displacement field to be captured, mostly from grain boundaries, and a fair interpolation of the displacement to be obtained within the grains. From these data, crystal rotations can be estimated very accurately. Both synthetic and real experimental cases are considered to illustrate the method.
\end{abstract}

Keyword: Digital Image Correlation, Displacement, EBSD, Regularization, Rotation

\section{Introduction}

During the last decades, many developments of multiscale plasticity models have been proposed. The motivation is often a better prediction of the macroscopic behavior by enriching the constitutive description with applications in metal forming or fatigue failure [20], or to estimate the long-term behavior of materials subjected to irradiation [25]. For industrial applications with these multiscale models, experimental validations are needed for each model and each scale transition.

The experimental analysis proposed in this paper is related to the scale transition from single crystal to macroscopic plasticity, which constitutes a homogenization problem. Mean-field homogenization is often analytical and for instance based on Eshelby's inclusion principle [5], while finite element calculations on polycristalline aggregates allow numerical homogenization to be performed together with the computation of local mechanical fields [3]. The main challenge of the scale transition between single crystal and polycrystals is to account for strain gradient contributions. Experimental assessments of strain gradient contributions have been proposed by comparing experiments involving grain boundaries with strain gradient crystal plasticity models [28] or by measuring the lattice curvature developing near grain boundaries using high angular resolution electron backscattered diffraction (HR-EBSD) [39]. On the other hand, strain gradients have a known influence on the material macroscopic response in plasticity and on the stresses developing at grain boundaries [9, 14]. In this context, several validations of crystal plasticity models exploiting kinematic fields have been conducted, and more specifically the displacement and strain fields obtained from images acquired with a scanning electron microscope (SEM) [21, 26].

Digital Image Correlation (DIC) techniques [32] have been used to compute kinematic fields from images registered at the microscale, for instance using SEM pictures [1, 10, 36, 33, 24]. It is worth noting that artifacts associated with the image capture in an SEM had to be accounted for [34, 30, 13]. DIC has also been utilized to characterize crystal deformations in processes such as fatigue and crack growth [6, 22]. In particular, EBSD images have been superimposed onto DIC strain maps to study the relationship between the texture and the plastic deformation at the crack tip [22]. Displacement fields only partly represent the kinematics of a polycrystal subjected to a mechanical loading. An important and complementary kinematics, when considering finite strain transformation, is lattice rotation. Measuring rotation fields can be achieved by using for instance orientation imaging microscopy based on HR-EBSD [17], or using synchrotron X-ray microdiffraction [23] providing average rotations per grain. 
Texture evolutions, measured using EBSD and grain averages, have already been used to validate crystal plasticity models [19] together with strain distributions in polycrystals.

In the following, it is proposed to simultaneously measure displacement and rotation fields by registering EBSD maps. Because of their sharp contrasts, grain boundaries allow for a good registration as will be shown below. However, one of the challenges is that intragranular deformation cannot be followed accurately as the orientation field shows very small gradients within the grains. Elastic regularization [31] is an efficient way to compensate for poorly contrasted images [38]. Spatial regularization is provided by a penalty to deviation of displacement fields from being the solution to a homogeneous linearly elastic problem, either isotropic, cubic or orthotropic. This procedure allows the displacements to be interpolated within the grains.

The paper is organized as follows. Section 2 is dedicated to the preparation of EBSD images for DIC analyses. It is proposed to use quaternions to encode orientation maps provided by EBSD analyses. The correlation algorithm based on orientation maps is developed in Section 3 to evaluate displacement fields. As a key result, the residual field indicates the disorientation, i.e., crystal rotations between images. Hence it is possible to measure displacement and crystal rotation fields. A numerical validation based on a synthetic example is first presented in Section 4 and an experimental application of the algorithm is detailed in Section 5.

\section{Quaternion-encoded orientation maps}

\subsection{General properties of quaternions}

Quaternions are hyper-complex numbers generalizing to four dimensions complex numbers in two dimensions [15, 16]. Quaternion algebra is a useful tool for the description of rotations and orientations in 3D spaces (akin to complex numbers for rotations and orientations in 2D). Quaternions are devoid of the singularity at the origin of Euler angle space, which is due to the progressive degeneracy of the first and third Euler angles as the second angle approaches zero (i.e., "gimbal lock"). Quaternions also provide a convenient frame to handle crystal symmetries [7].

Appendix A recalls important features of quaternions. Let us simply define here our notations. Quaternions consist of a real and three imaginary numbers $(1, \boldsymbol{i}, \boldsymbol{j}, \boldsymbol{k})$ so that any quaternion is described as $\boldsymbol{q}=q_{1}+q_{2} \boldsymbol{i}+q_{3} \boldsymbol{j}+q_{4} \boldsymbol{k}$, which is equivalent to the $4 \mathrm{D}$ vector representation $\boldsymbol{q}=\left(q_{1}, q_{2}, q_{3}, q_{4}\right)$. In the following, only unit quaternions

$|\boldsymbol{q}|^{2}=\sum_{n} q_{n}^{2}=1$ will be considered. A rotation of angle $\theta$ about an axis defined by a unit vector $\boldsymbol{d}=\left(d_{1}, d_{2}, d_{3}\right)$ is represented by the unit quaternion

$$
\begin{aligned}
\boldsymbol{q} & =e^{\frac{\theta}{2}\left(d_{1} \boldsymbol{i}+d_{2} \boldsymbol{j}+d_{3} \boldsymbol{k}\right)} \\
& =\cos (\theta / 2)+\left(d_{1} \boldsymbol{i}+d_{2} \boldsymbol{j}+d_{3} \boldsymbol{k}\right) \sin (\theta / 2)
\end{aligned}
$$

Equation (1) can be seen as an extension of Euler's formula.

Since a crystal orientation can be identified by the 3D rotation needed to bring a reference crystal orientation onto it, it can be described by a quaternion. As a consequence, the reference orientation is the unit real number $\boldsymbol{q}_{0}=(1,0,0,0)$. Successive applications of rotations $\boldsymbol{q}_{1}$ and $\boldsymbol{q}_{2}$ are obtained by a (non commutative) quaternion product $\boldsymbol{q}_{2} \times \boldsymbol{q}_{1}$ where the elementary multiplication rules are recalled in Appendix A. In the sequel the multiplicative sign $\times$ will be omitted to simplify notations. Thus the rotation that brings $\boldsymbol{q}_{1}$ onto $\boldsymbol{q}_{2}$ is $\boldsymbol{q}_{2} \boldsymbol{q}_{1}^{-1}$. A fundamental property that will be used in the sequel is that a "distance" between two orientations can be computed from the minimum magnitude of the disorientation angle $\theta$. The real part $\Re\left[\boldsymbol{q}_{2}^{-1} \boldsymbol{q}_{1}\right]=\cos (\theta / 2)$ gives the cosine of half the disorientation angle.

\subsection{Crystal symmetry and quaternions}

One practical difficulty associated with crystal symmetry is that several orientations having distinct quaternion representations are equivalent. For the cubic symmetry class, 48 quaternions $\boldsymbol{p}_{i}^{c u b}(1 \leq i \leq 48)$ are to be considered as identical to the reference orientation (Appendix B). When reading an orientation ".ang" file from an EBSD analysis re-encoding it as a field of quaternion requires a convention to be chosen in order to minimize spurious discontinuities due to arbitrary choices of quaternions within the symmetry class.

At a particular point of the EBSD map, where one determination of the orientation is converted into quaternion $\boldsymbol{q}(\boldsymbol{x})$, any other $\boldsymbol{p}_{n}^{c u b} \boldsymbol{q}(\boldsymbol{x})$ (for all $n$ ) is a valid choice. One possible convention is to select the one having the largest real part,

$$
\boldsymbol{q}^{(1)}(\boldsymbol{x})=\underset{n}{\operatorname{Argmax}}\left(\Re\left(\boldsymbol{p}_{n}^{c u b} \boldsymbol{q}(\boldsymbol{x})\right)\right)
$$


This choice can be understood as the one that requires the smallest rotation angle to be mapped onto the reference configuration i.e., $\boldsymbol{q}_{0}=1$. Figure 1 shows the quaternion field $\boldsymbol{q}^{(1)}(\boldsymbol{x})$ extracted from an $\mathrm{OIM}^{\mathrm{TM}}$ file. Some discontinuities observed in those maps do not correspond to a real grain boundary but simply to a slight rotation of the physical frame that produces a change in the symmetric representative.

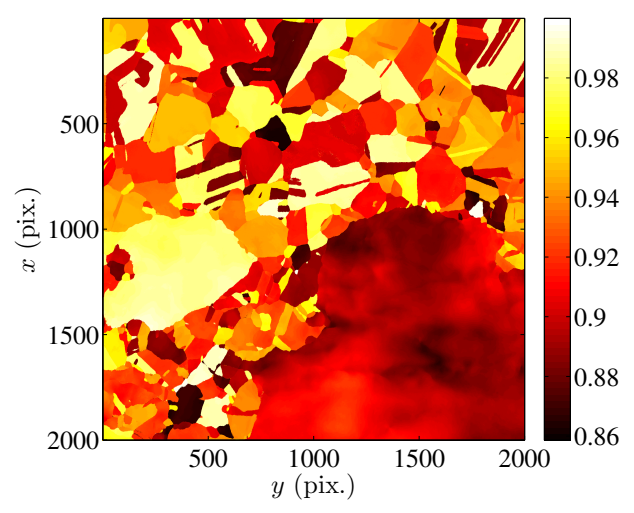

(a) $q_{1}$

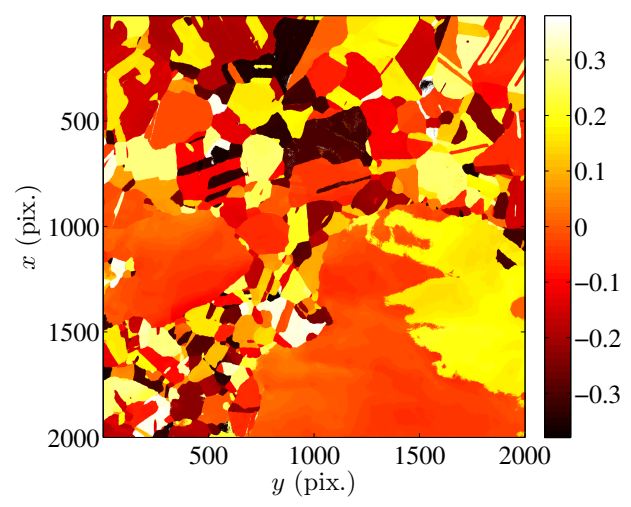

(c) $q_{3}$

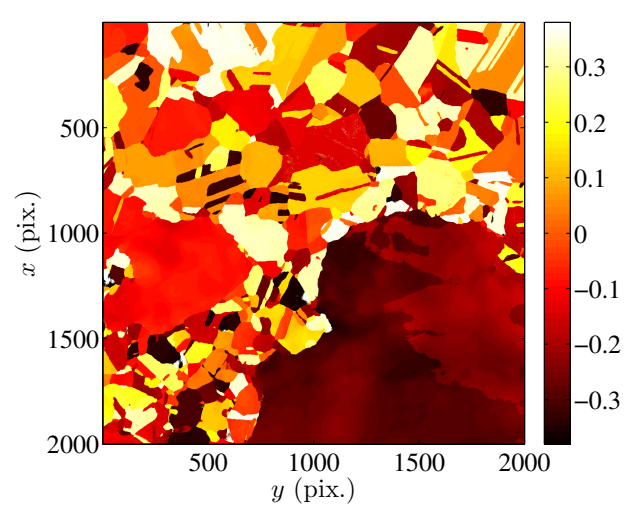

(b) $q_{2}$

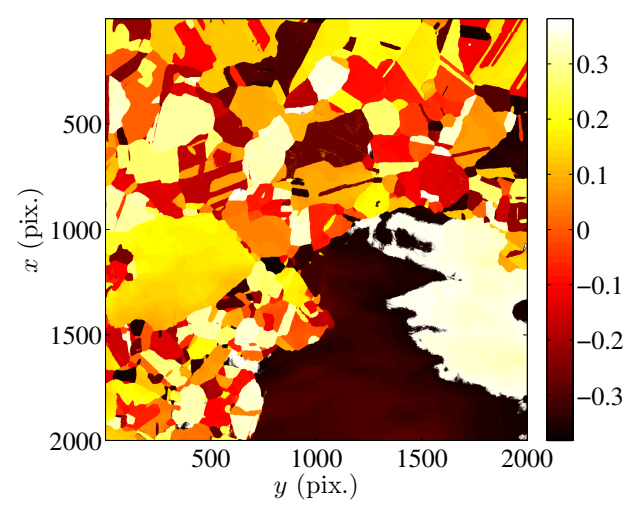

(d) $q_{4}$

Figure 1: Maps of the four components of the quaternion field after selecting the closest representative with respect to the reference orientation $\boldsymbol{q}_{0}=1$. The inverse pole figure corresponding to these maps is shown in Figure 10(b)

In order to reduce spurious discontinuities, a second procedure is proposed herein. After the first determination of orientation has been performed a local reference is defined per grain. It is chosen as the orientation at the center of mass of each grain. The labeling of grains is performed thanks to the OIM ${ }^{\mathrm{TM}}$ software, which provides the grain boundary image according to a given disorientation threshold, set to be 1 degree in practice. From this image, a label $\ell$ is assigned to each grain, and shared by all pixels $\boldsymbol{x}$ of the grain, so that one may refer to its label $\ell(\boldsymbol{x})$. The center of mass of the grain is also computed, $\boldsymbol{x}_{c}^{\ell}$. The local grain reference is then set to $\boldsymbol{q}^{r e f}(\boldsymbol{x})=\boldsymbol{q}^{(1)}\left(\boldsymbol{x}_{c}^{\ell(\boldsymbol{x})}\right)$. A new determination of the symmetric representative $\boldsymbol{q}^{(2)}(\boldsymbol{x})$ is performed by selecting the symmetric representative $n^{*}$ that maximizes the real part of $\boldsymbol{p}_{n}^{c u b} \boldsymbol{q}^{r e f}(\boldsymbol{x})^{-1} \boldsymbol{q}(\boldsymbol{x})$, i.e., choosing the orientation that is the nearest to that of the center of mass of the grain. Figure 2 shows the resulting quaternion map. This second convention will reveal very convenient for filtering quaternion fields, which will be detailed in Section 3.2. Its justification is that within one grain, the change in orientation is small, and hence, no spurious apparent discontinuities will appear.

\section{Proposed correlation algorithm}

Conventional DIC consists in registering two gray-level images, one in the reference state $f(\boldsymbol{x})$ and a second one in the deformed state $g(\boldsymbol{x})$. It is based on the determination of the displacement field $\boldsymbol{u}(\boldsymbol{x})$ such that the corrected deformed image $\widetilde{g}_{\boldsymbol{u}}(\boldsymbol{x})=g(\boldsymbol{x}+\boldsymbol{u}(\boldsymbol{x}))$ coincides as best as possible with the reference image $\widetilde{g}_{\boldsymbol{u}}(\boldsymbol{x})=f(\boldsymbol{x})$, and hence minimizes the quadratic norm of the so-called residual $\left(\widetilde{g}_{\boldsymbol{u}}(\boldsymbol{x})-f(\boldsymbol{x})\right)$ summed over the entire region of interest.

The proposed extension consists in matching quaternion-valued images $\boldsymbol{f}(\boldsymbol{x})$ and $\boldsymbol{g}(\boldsymbol{x})$. Hence, the crystallographic orientation is treated as equivalent to a speckle pattern, as the orientation is not expected to undergo large 


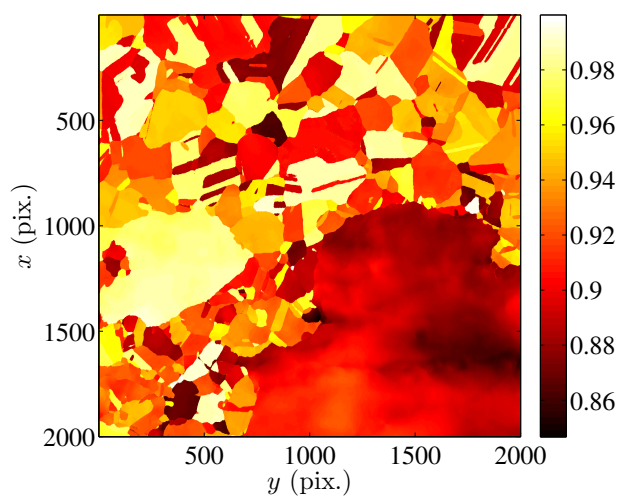

(a) $q_{1}$

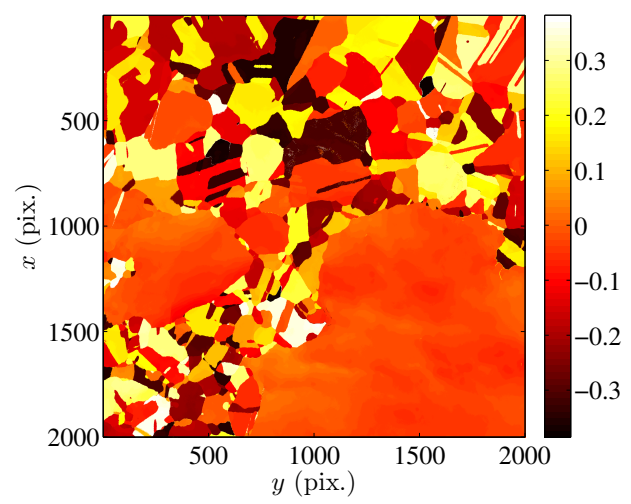

(c) $q_{3}$

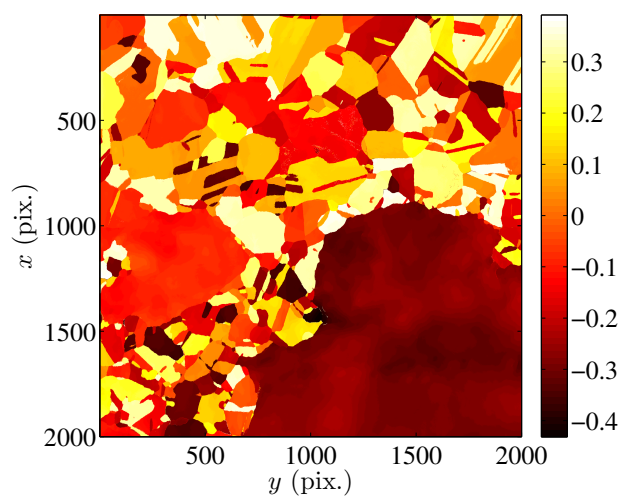

(b) $q_{2}$

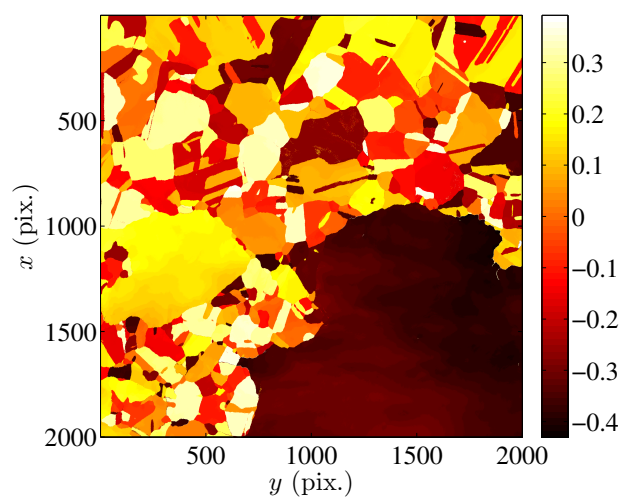

(d) $q_{4}$

Figure 2: Maps of the four components of the quaternion field after selection of the closest representative to the local grain reference $\boldsymbol{q}^{r e f}(\boldsymbol{x})$. The inverse pole figure corresponding to these maps is shown in Figure 10(b)

changes during deformation. Following the above schematic formulation, a corrected deformed image is introduced $\widetilde{\boldsymbol{g}}_{\boldsymbol{u}}(\boldsymbol{x})=\boldsymbol{g}(\boldsymbol{x}+\boldsymbol{u}(\boldsymbol{x}))$, and the registration is achieved via the minimization of the "discrepancy" defined here as

$$
\Phi[\boldsymbol{u}]=\sum_{R O I} \psi^{2}\left(\widetilde{\boldsymbol{g}}_{\boldsymbol{u}}(\boldsymbol{x}), \boldsymbol{f}(\boldsymbol{x})\right)
$$

so that the sought displacement field is the minimizer of $\Phi$

$$
\boldsymbol{u}(\boldsymbol{x})=\underset{\boldsymbol{u}}{\operatorname{Argmin}} \Phi[\boldsymbol{u}]
$$

In order to follow a strict parallel with classical DIC, $\psi$ should measure a distance in unit quaternion space between its two arguments. As above discussed, a good definition for this "distance" is based on the minimum magnitude of the angle of the rotation $\theta$ relating the two orientations. In addition, all possible symmetry replicas of one of the two arguments should be considered and the minimum value should be selected. This operation is numerically expensive as it has to be performed pixelwise. Considering that the discrepancy between orientations will be small at convergence, it is proposed to substitute to the above quadratic distance a function that behaves similarly close to the origin, namely $1-\cos (\theta / 2) \sim_{\theta \rightarrow 0} \theta^{2} / 8$. Thus, assuming crystal symmetry degeneracies are accounted for, the following choice of $\psi^{2}$ is made

$$
\begin{aligned}
\psi^{2}(\boldsymbol{f}, \boldsymbol{g}) & \equiv \Re\left[1-\boldsymbol{f g}^{-1}\right] \\
& =1-f_{i} g_{i} \\
& =1-\cos (\theta / 2)
\end{aligned}
$$

where $\Re$ denotes the real part (or first component) of the quaternion. Last, without further restriction, the problem is generally ill-posed. Thus following the usual practices of DIC, the displacement field is sought as the minimizer of $\Phi[\boldsymbol{u}]$ where $\boldsymbol{u}$ is confined to a vector space generated by a suited basis of vector fields $\boldsymbol{\varphi}_{i}$, so that

$$
\boldsymbol{u}(\boldsymbol{x})=\sum_{i=1}^{m} \lambda_{i} \boldsymbol{\varphi}_{i}(\boldsymbol{x})
$$


This formulation leaves $\lambda_{i}$ as unknowns that can be determined through a Gauss-Newton algorithm [27].

\subsection{Gauss-Newton scheme}

A Gauss-Newton procedure iteratively computes corrections $\left\{\boldsymbol{\delta} \boldsymbol{\lambda}^{(n)}\right\}$ to the column vector $\left\{\boldsymbol{\lambda}^{(n-1)}\right\}$, based on the solution to a linearized system

$$
\left[\boldsymbol{M}^{(n-1)}\right]\left\{\boldsymbol{\delta} \boldsymbol{\lambda}^{(n)}\right\}=\left\{\boldsymbol{\gamma}^{(n-1)}\right\}
$$

where $\left[\boldsymbol{M}^{(n-1)}\right]$ and $\left\{\boldsymbol{\Gamma}^{(n-1)}\right\}$ are respectively the approximate Hessian matrix and residual vector that are to be updated at each iteration $n$. The correction vector is $\boldsymbol{\lambda}^{(n)}=\boldsymbol{\lambda}^{(n-1)}+\boldsymbol{\delta} \boldsymbol{\lambda}^{(n)}$.

A reformulation based on quaternion algebra is given in this subsection. If the reader is interested in the final results, intuitive and familiar expressions of both Jacobian $\left\{\gamma^{(n-1)}\right\}$ and Hessian $[\boldsymbol{M}]$ are retrieved in Equations (14) and (15) respectively. The Jacobian reads

$$
\begin{aligned}
\gamma_{i}^{(n)} & \equiv \frac{\partial \Phi}{\partial \lambda_{i}} \\
& =-\sum_{R O I} \Re\left[\boldsymbol{f}(\boldsymbol{x}) \boldsymbol{\nabla}\left(\widetilde{\boldsymbol{g}}^{(n)}(\boldsymbol{x})\right)^{-1}\right] \boldsymbol{\varphi}_{i}(\boldsymbol{x}) \\
& =-\sum_{R O I}\left(\sum_{\alpha} f_{\alpha}(\boldsymbol{x}) \boldsymbol{\nabla} \tilde{g}_{\alpha}^{(n)}(\boldsymbol{x})\right) \boldsymbol{\varphi}_{i}(\boldsymbol{x})
\end{aligned}
$$

and the Hessian

$$
\begin{aligned}
M_{i j}^{(n-1)} & \equiv \frac{\partial^{2} \Phi}{\partial \lambda_{i} \partial \lambda_{j}} \\
& =-\sum_{R O I} \Re\left[\boldsymbol{f}(\boldsymbol{x})(\boldsymbol{\nabla} \otimes \boldsymbol{\nabla})\left(\widetilde{\boldsymbol{g}}^{(n-1)}(\boldsymbol{x})\right)^{-1}\right]:\left(\boldsymbol{\varphi}_{i}(\boldsymbol{x}) \otimes \boldsymbol{\varphi}_{j}(\boldsymbol{x})\right) \\
& =-\sum_{R O I}\left(\sum_{\alpha} f_{\alpha}(\boldsymbol{x})(\boldsymbol{\nabla} \otimes \boldsymbol{\nabla}) \tilde{g}_{\alpha}^{(n-1)}(\boldsymbol{x})\right):\left(\boldsymbol{\varphi}_{i}(\boldsymbol{x}) \otimes \boldsymbol{\varphi}_{j}(\boldsymbol{x})\right)
\end{aligned}
$$

Since quaternions can be seen as unit vectors in a $4 \mathrm{D}$ space, they obey $\boldsymbol{q q}^{-1}=q_{\alpha} q_{\alpha}=1$ with $\alpha=1, \ldots, 4$. Therefore they are always orthogonal to their gradient

$$
\boldsymbol{\nabla}\left(\boldsymbol{q} \boldsymbol{q}^{-1}\right)=\mathbf{0}
$$

and in particular for the real part of this identity, $q_{\alpha} \boldsymbol{\nabla} q_{\alpha}=0$

$$
(\boldsymbol{\nabla} \otimes \nabla)\left(\boldsymbol{q} \boldsymbol{q}^{-1}\right)=\mathbf{0}
$$

and thus in particular, $q_{\alpha}(\boldsymbol{\nabla} \otimes \boldsymbol{\nabla}) q_{\alpha}+\boldsymbol{\nabla} q_{\alpha} \otimes \nabla q_{\alpha}=\mathbf{0}$. The last identity is used to rewrite second order derivatives as

$$
q_{\alpha}(\nabla \otimes \nabla) q_{\alpha}=-\nabla q_{\alpha} \otimes \nabla q_{\alpha}
$$

or $f_{\alpha} f_{\alpha, i j}=-f_{\alpha, i} f_{\alpha, j}$.

Using the above properties leads to equivalent expressions of the residual vector $\left\{\boldsymbol{\gamma}^{(n-1)}\right\}$ and Hessian $\left[\boldsymbol{M}^{(n-1)}\right]$. The former is rewritten using Equation (10)

$$
\gamma_{i}^{(n-1)}=-\sum_{R O I}\left(f_{\alpha}(\boldsymbol{x})-\tilde{g}_{\alpha}^{(n-1)}\right) \nabla \tilde{g}_{\alpha}^{(n-1)}(\boldsymbol{x}) \cdot \boldsymbol{\varphi}_{i}(\boldsymbol{x})
$$

Moreover since $\widetilde{\boldsymbol{g}}^{(n-1)}$ is expected to converge to $\boldsymbol{f}$, one may substitute one to the other at dominant order and finally express $\gamma$ as

$$
\gamma_{i}^{(n-1)}=\sum_{R O I}\left(\nabla f_{\alpha}(\boldsymbol{x}) \cdot \boldsymbol{\varphi}_{i}(\boldsymbol{x})\right)\left(\tilde{g}_{\alpha}^{(n-1)}-f_{\alpha}(\boldsymbol{x})\right)
$$

The same simplification can be extended to the Hessian. Equation (12) is used to transform the second order derivatives into first order ones, and again $\boldsymbol{f}$ is substituted to $\widetilde{\boldsymbol{g}}^{(n-1)}$

$$
M_{i j}=\sum_{R O I}\left(\nabla f_{\alpha}(\boldsymbol{x}) \cdot \boldsymbol{\varphi}_{i}(\boldsymbol{x})\right)\left(\boldsymbol{\nabla} f_{\alpha}(\boldsymbol{x}) \cdot \boldsymbol{\varphi}_{j}(\boldsymbol{x})\right)
$$


Let us emphasize that the final expression of $[\boldsymbol{M}]$ does not depend any longer on the iteration number $n$, and hence it can be computed once for all. Although the above detailed justification appears to be somewhat involved, the final expressions of $[\boldsymbol{M}]$ and $\boldsymbol{\gamma}^{(n-1)}$ could have been guessed by extending global DIC to quaternions componentwise. For a single $\alpha$, the expression of $[\boldsymbol{M}]$ and $\gamma^{(n-1)}$ are exactly those of global DIC [18], where the quaternion component $f_{\alpha}$ is analogous to the gray level of a speckle pattern. Thus the summation over the four components is equivalent to having four different speckles (i.e., four imaging modalities) of the same surface, which would all obey the same kinematics. A parallel could be drawn with DIC based on color images, wherein each channel R, G or B would be treated as an independent information.

\subsection{Filtering quaternion fields}

One difficulty is related to the discontinuities of $\boldsymbol{f}$ (and $\boldsymbol{g}$ ). The orientation is uniform in each grain but each grain boundary appears as a large discontinuity. This specific texture (i.e., piecewise constant texture) is not very well suited to a Gauss-Newton scheme as gradients do not give a fair picture of how $\boldsymbol{f}$ varies with the displacement over large distances. In order to be able to correct for large displacements, a solution consists of smoothing out the grain boundaries. A simple way to achieve such filtering is to apply to the quaternion field a convolution, component-wise, with a Gaussian kernel of width $\xi$, followed by a reprojection on the unit sphere in $4 \mathrm{D}$ (to preserve the unitary property of quaternions that has been lost in the convolution). Such filtered quaternion field is denoted as $\widehat{\boldsymbol{f}_{\xi}}$.

For given length $\xi$ the correlation procedure is straightforward as above discussed. The only care to be taken is that $\boldsymbol{g}$ should be first corrected by the displacement field, using the raw signal, and then filtered at the scale $\xi$, in order to compute differences with the filtered reference image. This determination tolerates displacement amplitudes of order $\xi$. Hence a large $\xi$ is to be used to initialize the procedure. However, the accuracy of the evaluation of the displacement is based on the sharpness of the grain boundary. A smooth grain boundary may only provide a gross determination. Hence it is proposed to progressively reduce the length $\xi$ using the previously measured displacement field at a large $\xi$ value to initialize a computation with a smaller $\xi$.

\subsection{Elastic regularization}

EBSD images generally show high contrast only between grains (see e.g., Figures 1 and 2) thus the correlation procedure determines the displacement field at grain boundaries. Seeking the displacement field inside grains by DIC is not a well-posed question, even if the displacement field is considered in a weak formulation as shown in Equation (6). An elastic regularization provides a very convenient interpolation scheme for the displacement field inside the grains where the image contrast is insufficient for registration purposes.

Mechanics-aided DIC has been proposed to solve such issues [31, 38], and the regularization technique has also been applied to digital volume correlation [35]. Readers are referred to these works for detailed justification. The spirit of the approach, which was pioneered by Tikhonov and Arsenin [37], is to introduce a regularization term, i.e., an additional functional of the displacement field that incorporates a prior information (or assumption) on the displacement field. However, one would like the regularization to interfere in a minimal way with the field to be measured, minimal meaning that it should matter only when no other data can be exploited. The chosen reference is to favor locally solutions to homogeneous elasticity, without specifying boundary conditions. The "equilibrium gap" is precisely one such functional, based on a second order differential operator acting on the displacement, and which is quadratic in the displacement [31,2]. It can be supplemented by a similar edge regularization (otherwise absent from the bulk equilibrium gap) [38].

Two parameters are used to characterize the weight of mechanical regularization, i.e., $\ell_{b}$ and $\ell_{m}$, representing the regularization lengths at the edge of the ROI and in the bulk of the ROI respectively. According to previous works [38], the optimized ratio $\ell_{b} / \ell_{m}=0.5$ is chosen for all the calculations reported hereafter. It should be noted that due to the contrast being concentrated at grain boundaries, the minimal mechanical regularization length $\ell_{m}$ should at least be of the same order of magnitude as the grain size to provide a mechanical regularization in the core of the grains without constraining too much the displacement determined at grain boundaries.

\subsection{Correlation residuals}

When registering EBSD images, it is expected that the motions of grain boundaries are mainly captured. Within each grain, elastic regularization will provide a smooth interpolation that is unbiased by the superposition of arbitrary rigid body motion, or even any displacement field that would be the solution to a homogeneous linear elastic problem. While displacement accuracy is reduced far from grain boundaries, disorientations are expected to be small in these areas, and therefore the residual will allow the crystal rotations to be quantified very accurately. 
Further, at the grain boundaries the confidence index and image quality of EBSD acquisition tend to be low. If the electron beam hits two grains at the same time an unreliable orientation will be recorded. Besides, at grain boundaries the interpolation procedure of $\boldsymbol{g}$ results in an orientation that is close to neither grain. Therefore grain boundaries will always appear distinctively in the residual fields.

\section{Application of the algorithm on a synthetic test case}

A synthetic test case is numerically generated in order to validate the determination of the displacement field with known values. The displacement field is computed from a finite element simulation with Code_Aster [8]. A twodimensional microstructure shown in Figure 3 with isotropic texture is modeled from an experimental orientation map.

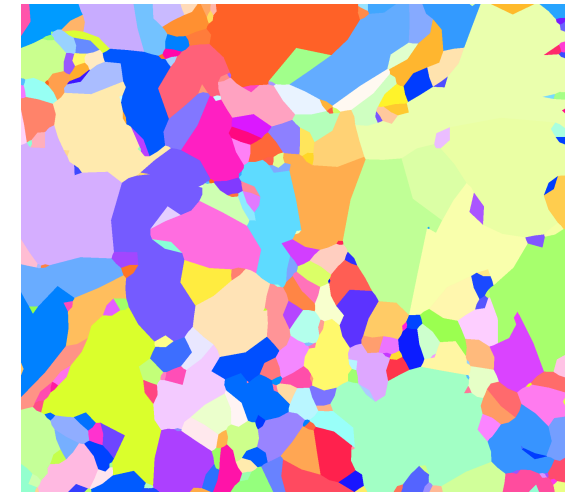

(a)

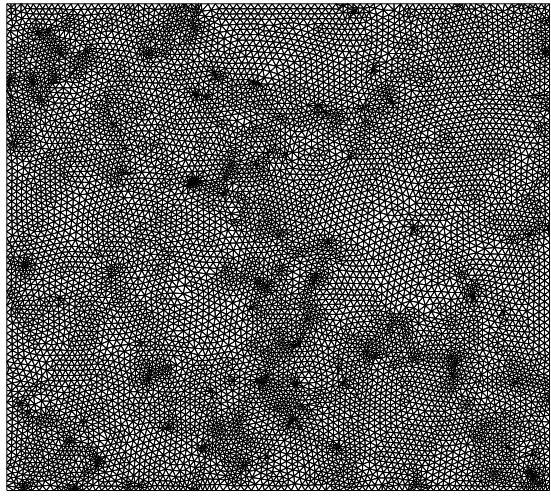

(c)

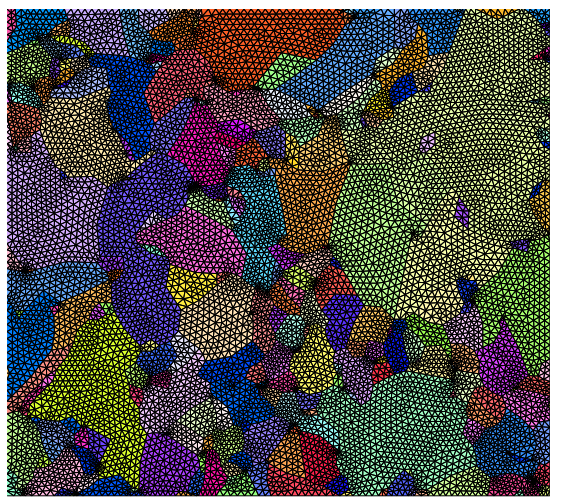

(d)

Figure 3: (a) Inverse pole figure of the 2D polycrystalline model for FE simulations. (b) FE mesh utilized in tensile simulation. (c) FE mesh superimposed onto the polycrystalline model

It allows a mesh to be created in such a way that it very closely follows the grain boundaries, (GB), as performed in Refs. $[12,11,4]$. The ability for the mesh to conform with GBs opens the way to detect and measure strain discontinuities across GBs. This property holds for FE-based (global) DIC when a speckle pattern marking is deposited on the observed surface. It also holds herein since the GBs carry the information exploited by quaternion correlation, namely, the component of velocity normal to GBs. This property is valuable as any other approach would either betray the grain geometry or determine displacements only away from GBs.

The finite element calculation is performed under a plane strain assumption. Crystallographic orientations are uniform inside each grain. The numerical model is subjected to a monotonic uniaxial tensile simulation up to $6 \%$ of macroscopic strain. A constitutive law based on dislocation dynamics for body centered cubic crystals (DD_CC [29]) has been chosen in the FE simulation. A finite strain framework is used to implement the constitutive equations in order to properly account for the rotation of crystals during the tensile loading. The displacement field and crystal rotation in the ROI obtained by the FE simulation are shown in Figure 4.

Virtual EBSD images are processed for different macroscopic strain levels as shown in Figure 5. The displacement and crystal orientation at the nodes of the model are updated by FE simulation, and the orientation at virtual 


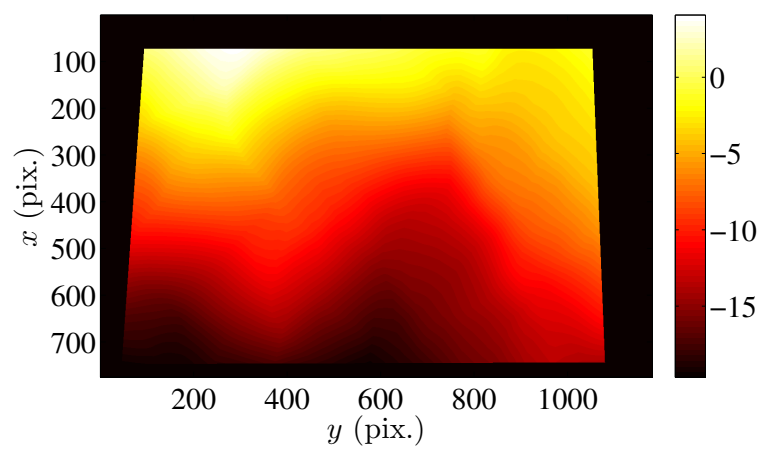

(a) $u_{x}$ (pixels)

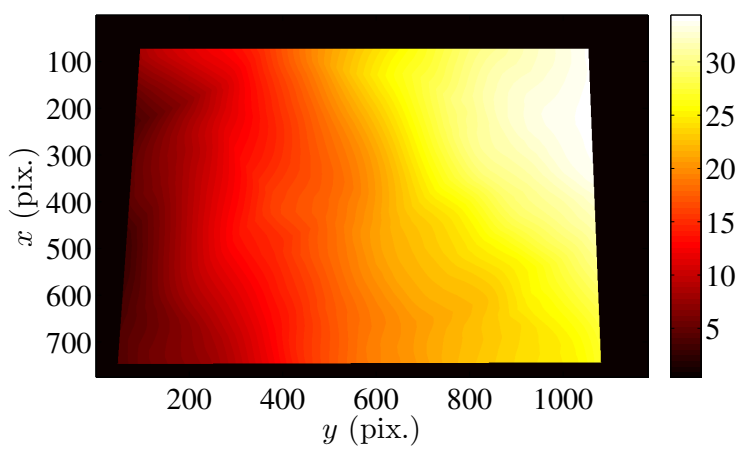

(b) $u_{y}$ (pixels)

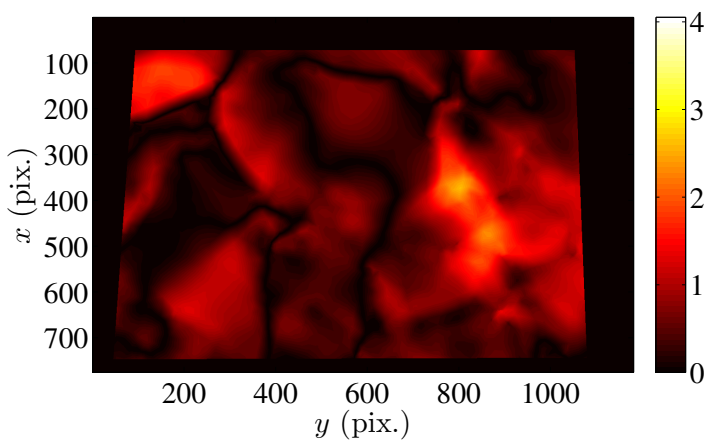

(c) $\theta$ (degrees)

Figure 4: Displacement (a-b) and crystal rotation $\theta$ (c) fields given by FE simulation

acquisition points inside each element are obtained by linear interpolation (see Appendix A) from the data of the three vertices (i.e., corner nodes). Therefore, the orientation at each acquisition point is always affected by the crystal orientation of only one grain, resulting in distinct grain boundaries. It should be noted that this is an ideal scenario that does not exist in realistic EBSD acquisition. A correlation analysis is performed with the orientation maps (Figure 3(a) and 5(a-c)). The calculation is run with a Matlab ${ }^{\mathrm{TM}}$ implementation on a computer with an i7 CPU.

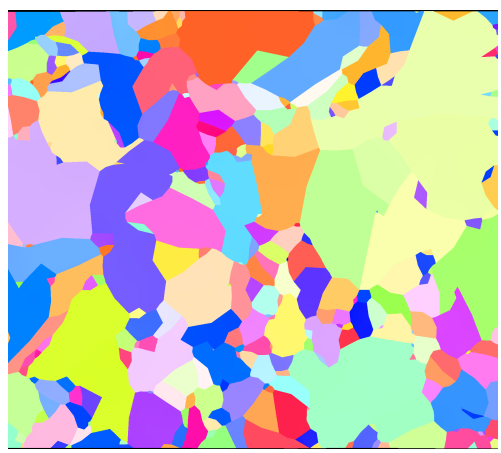

(a) $0.6 \%$

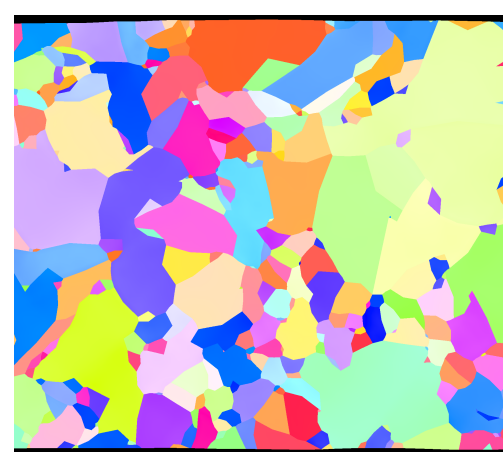

(b) $3 \%$

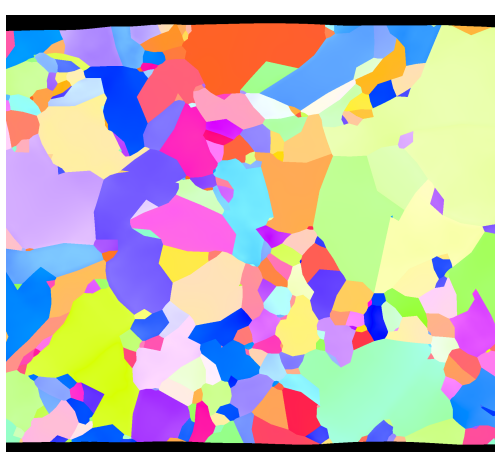

(c) $6 \%$

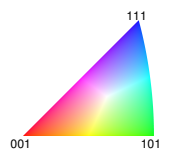

Figure 5: Inverse pole figures corresponding to macroscopic strain equal to $0.6 \%$ (a), $3 \%$ (b) and $6 \%$ (c) respectively

The analyzed ROI has a size of $775 \times 1181$ pixels (Figure $6(\mathrm{a})$ ). The registration procedure is performed twice, namely, on two different meshes consisting of triangular elements with a characteristic length of 50 pixels and 10 pixels respectively (Figure 6(b-c)). It should be noted that the meshes are not conforming to the grain geometry. 


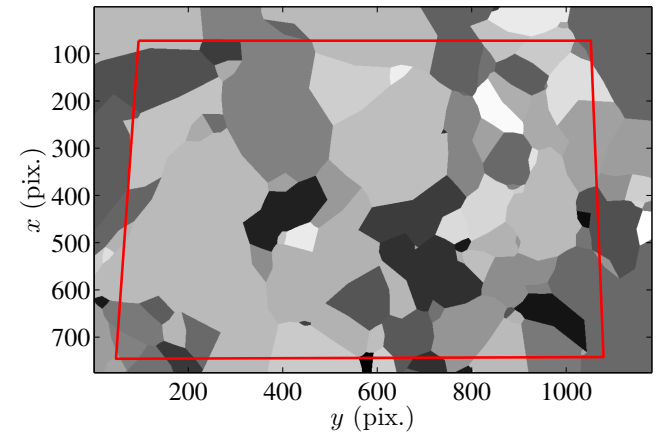

(a)

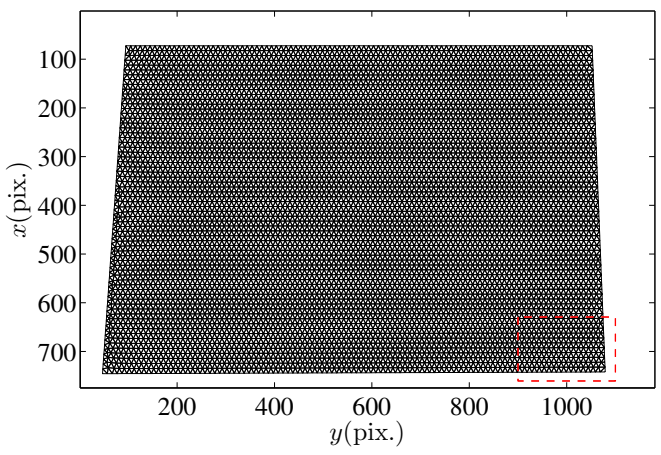

(c)

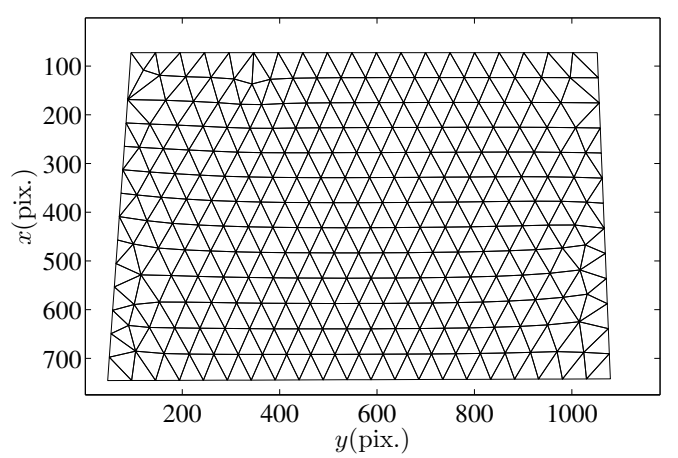

(b)

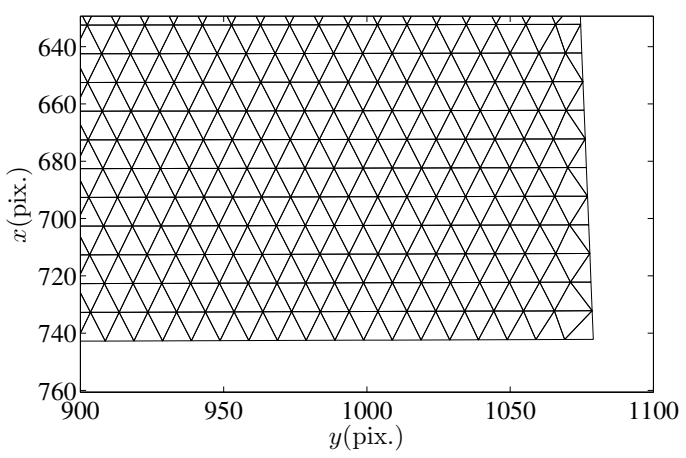

(d)

Figure 6: Parameters used in quaternion correlation of the validation test case. (a) ROI of the artificial reference EBSD image. (b) FE mesh with characteristic length of 50 pixels. (c) FE mesh with characteristic length of 10 pixels. The boxed zone is zoomed in $(\mathrm{d})$

When one Gauss-Newton iteration results in an average incremental displacement less than $5 \times 10^{-5}$ pixel, convergence is obtained. To avoid local minimum trapping, the elastic regularization length $\ell_{m}$ and the length of the Gaussian kernel $\xi$ are both initialized with high values. Once convergence is reached, the resulting displacement field is used to initialize the next correlation step in which the values of $\ell_{m}$ and $\xi$ gradually decrease. Table 1 gives an example of the settings of quaternion correlation for the final state of $\ell_{m}=100$ pixels and $\xi=0$. It should be noted that $\xi=0$ means that the quaternion field is not subjected to any filtering. In total, about 700 iterations are needed to reach convergence.

Table 1: Evolution of $\ell_{m}$ and $\xi$ during quaternion correlation

\begin{tabular}{rrrrrrrr}
\hline Step & 1 & 2 & 3 & 4 & 5 & 6 & 7 \\
\hline$\ell_{m}$ (pixel) & 400 & 400 & 400 & 200 & 100 & 100 & 100 \\
\hline$\xi$ (pixel) & 16 & 3 & 2 & 2 & 2 & 1.2 & 0 \\
\hline Number of iterations & 484 & 68 & 22 & 41 & 61 & 43 & 10 \\
\hline
\end{tabular}

The displacement error is computed from the root mean square (RMS) difference between computed and measured vector displacement fields. In order to optimize the parameterization of mechanical regularization $\ell_{m}$, the displacement error obtained with the mesh with characteristic length of 50 pixels is shown in Figure 7 (a) for different values of $\ell_{m}$ and three different macroscopic strains, $0.6 \%, 3 \%$ and $6 \%$. The error tends to be high for large $\ell_{m}$ values (200 pixels) as for such sizes, the regularization influences the registration of grain boundaries. The error displays small variations at different macroscopic strain levels in case of small $\ell_{m}$ levels (50 pixels) as the displacement field around the center of grains is not regularized by the orientation contrast at grain boundaries. Therefore the displacement error is higher inside grains. The best compromise is obtained when $\ell_{m}=100$ pixels. This is in agreement with the fact that the equivalent grain size in the ROI is 85 pixels as shown in Figure 7(b), 
and hence this size is a target value for $\ell_{m}$. It is to be noted however that the grain size is heterogeneous, and hence selecting a single value for the entire ROI results from a compromise. Let us finally note that the error tends to increase with the macroscopic strain, presumably because the effect of regularization on the displacement increases with the latter, and the elastic regularization becomes poorer and poorer adapted as the mean strain increases.

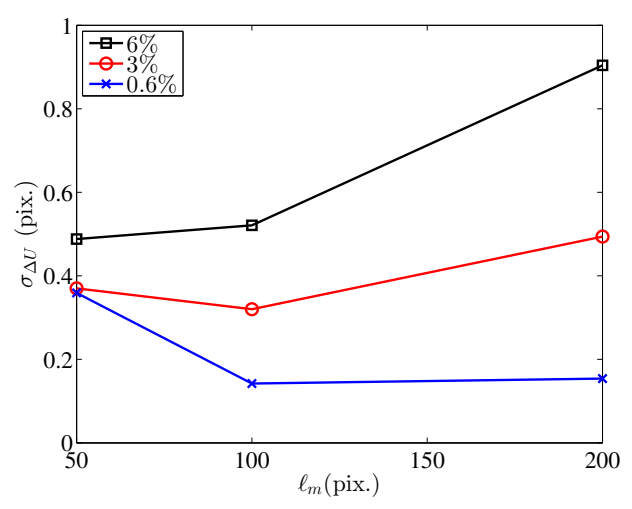

(a)

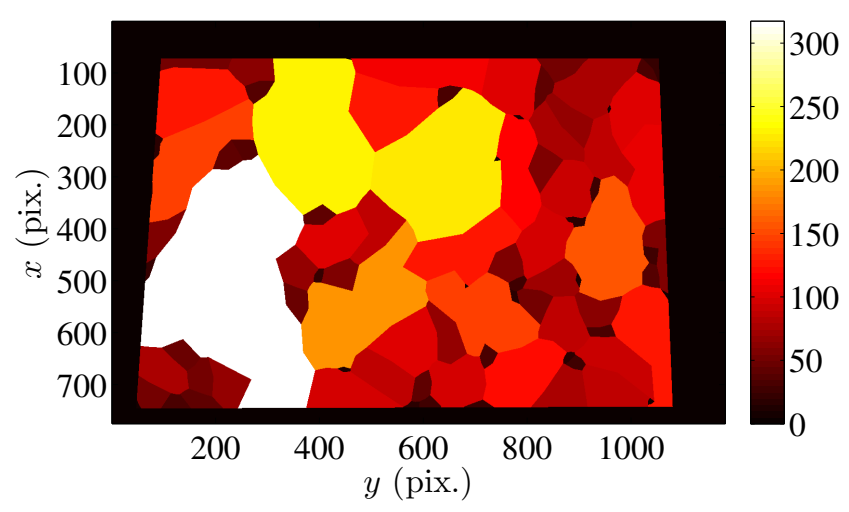

(b)

Figure 7: (a) RMS displacement error as a function of $\ell_{m}$ for different macroscopic strain levels: $0.6 \%, 3 \%$ and $6 \%$. Results with 50-pixel characteristic length mesh. (b) Equivalent grain size in ROI. The mean equivalent grain size is 85 pixels in the ROI

The performances of calculations on meshes of different sizes are listed in Table 2. It is seen that the error of displacement and rotation measurements is slightly lower with finer mesh elements, whereas the computation time is significantly longer.

Table 2: Performance of quaternion correlation for meshes of different sizes for a macroscopic strain of $3 \%$ and $\ell_{m}=100$ pixels

\begin{tabular}{cccc}
\hline Characteristic length & iteration time & RMS of $\Delta U$ & RMS of intra-grain $\Delta \theta$ \\
\hline 50 pixels & $17 \mathrm{~s}$ & 0.320 pixel & 0.0026 degree \\
\hline 10 pixels & $135 \mathrm{~s}$ & 0.305 pixel & 0.0024 degree \\
\hline
\end{tabular}

The quaternion correlation results between the initial state and $3 \%$ strain with $\ell_{m}=100$ pixels and 10-pixel elements are illustrated in Figure 8. At convergence, the residual, $\left[\boldsymbol{f} \widetilde{\boldsymbol{g}}^{-1}\right]$, is the disorientation between the reference and the deformed orientation transported with the measured displacement field. This quaternion field can be decomposed into a disorientation angle $\theta, 2 \times \cos ^{-1}\left(\Re\left[\boldsymbol{f} \widetilde{\boldsymbol{g}}^{-1}\right]\right)$, shown in Figure $8(\mathrm{a})$, and the orientation of the rotation direction $\left(v_{x}, v_{y}, v_{z}\right)$ (i.e., imaginary components of $\left[\boldsymbol{f} \widetilde{\boldsymbol{g}}^{-1}\right]$ rescaled to a unit norm), shown in Figure 8(bd). The disorientation angle remains quite small (typically from 1 to 2 degrees) and is markedly affected by the crystallographic orientation. Some grains are prone to displaying lattice rotations whereas others are not. It is observed from Figure 8(b-d) that the rotation axis, roughly uniform within grains, tends to be close to the $z$-axis. This is consistent with the plane-strain setting used in the FE simulation.

The measured displacement field is shown in Figure 8(e-f) and the differences between the measured and computed displacement fields are shown in Figure $8(\mathrm{~g}-\mathrm{h})$. The texture is no longer visible on the two images, which means that there is no noticeable difference for displacements at grain boundary and within grains. The RMS of displacement error reaches 0.3 pixel, which is high compared with the error of standard DIC (usually of the order of 0.01 pixel). This difference is attributed to the complete absence of contrast inside the grains, and the difficulty to set a fair interpolation of the orientation very close to grain boundaries. Figure 6(a) shows the used texture that is not the best suited to DIC, and even dealing with a scalar gray level would not allow such low uncertainty values to be reached. On the contrary, it is noteworthy that in spite of such poor texture, the resulting displacement difference is still well below 1 pixel.

The disorientation angle is shown in Figure 8(a). It should however not be considered as an error. In the simulation grains do rotate during their plastic deformation, and hence this phenomenon violates the assumption that the crystallographic orientation remains invariant. Because this rotation is small, it does not prevent the correlation 


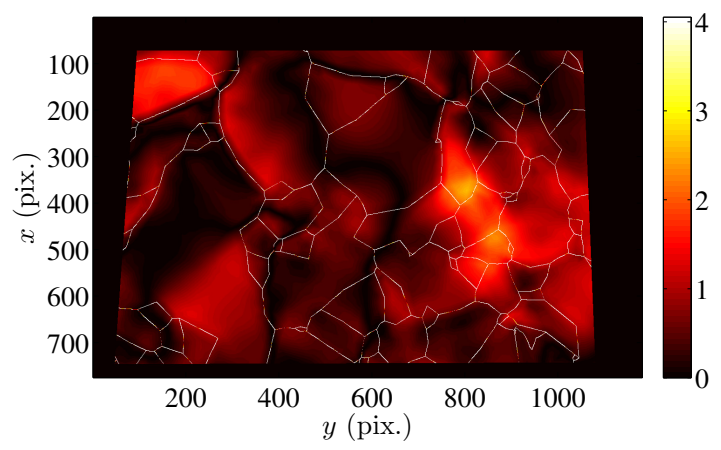

(a) $\theta$ (degrees)

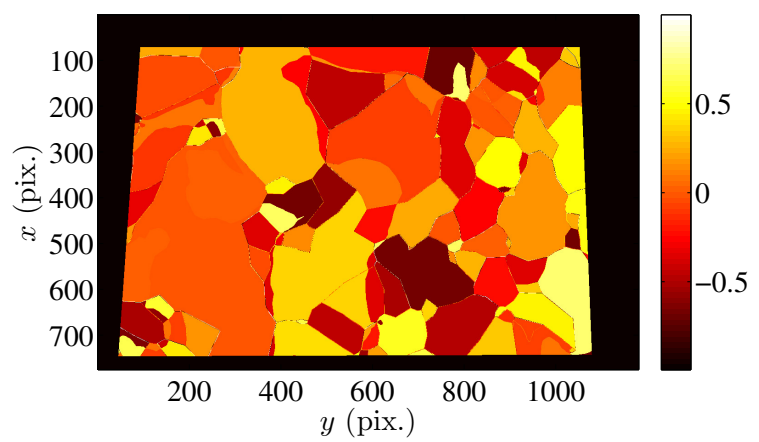

(c) $v_{y}$

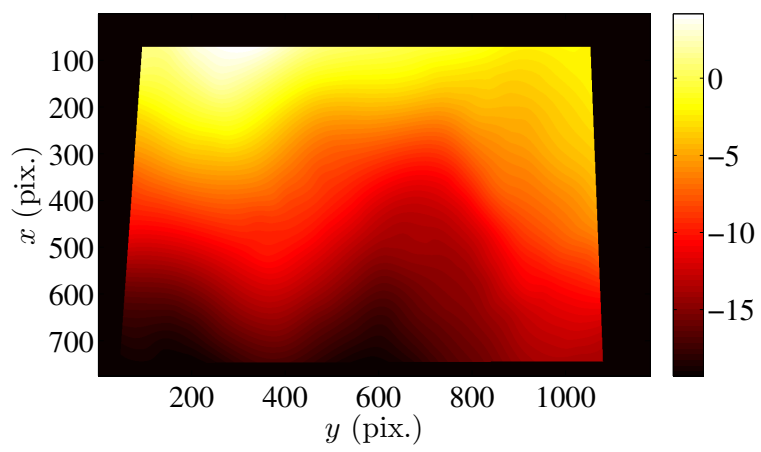

(e) $u_{x}$ (pixels)

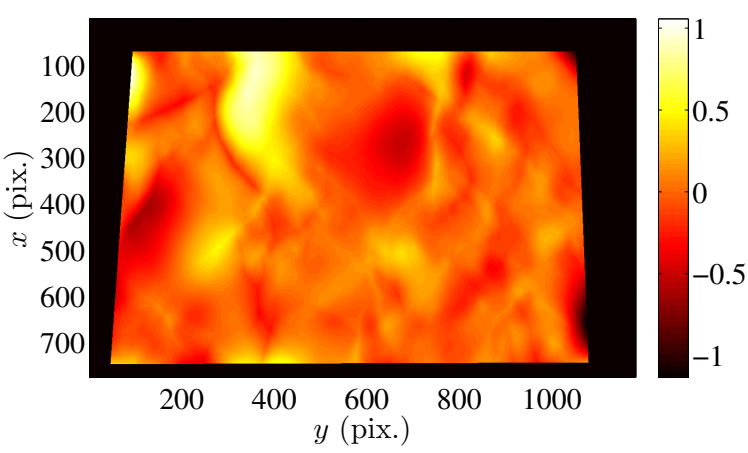

(g) $\Delta u_{x}$ (pixels)

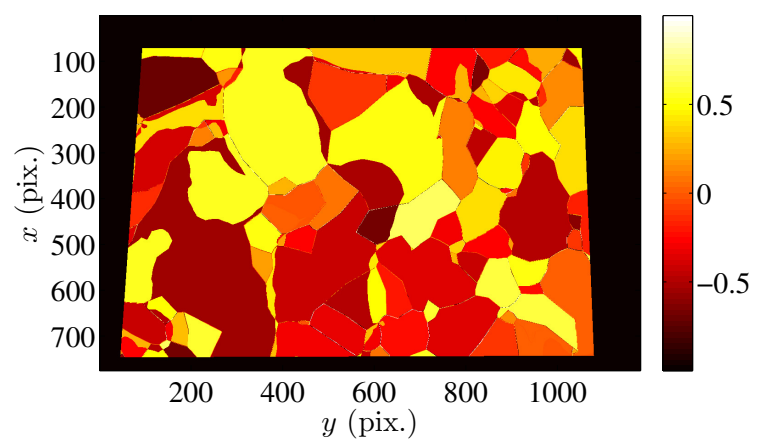

(b) $v_{x}$

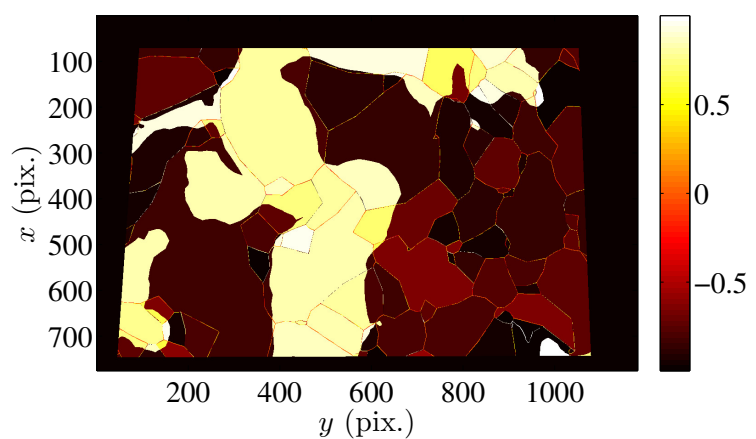

(d) $v_{z}$

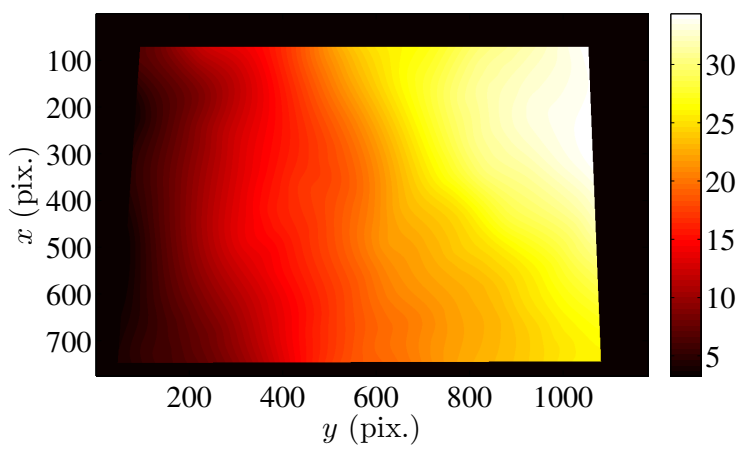

(f) $u_{y}$ (pixels)

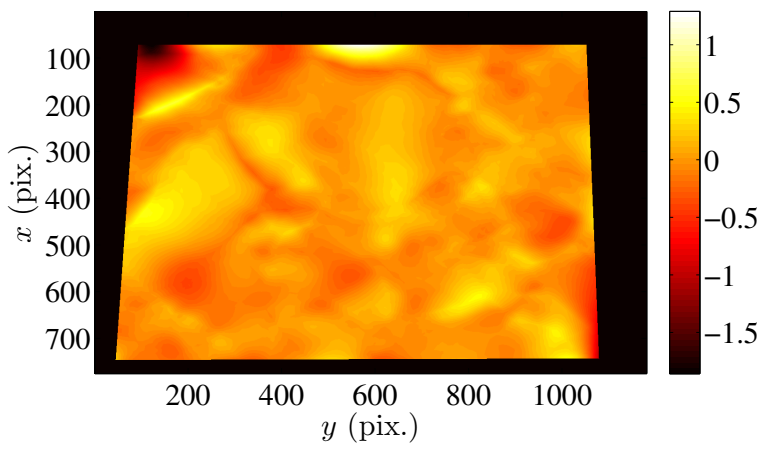

(h) $\Delta u_{y}$ (pixels)

Figure 8: Quaternion correlation residual disorientation decomposed into a disorientation angle (a), and the three components of the rotation director (b-d). Measured displacement field along the $x$ (e) and $y$ (f) directions, and the displacement difference along the $x(\mathrm{~g})$ and $y(\mathrm{~h})$ directions. Results obtained with $\ell_{m}=100$ pixels, 10-pixel elements 
procedure to extract the displacement field. This disorientation angle can be compared with the computed one. The difference using the same scale as Figure 8(a) is shown in Figure 9(a). It is observed that the difference is mostly localized at grain boundaries (for reasons discussed in Section 3.4). Figure 9(b) shows the error of rotation measurement inside the grains, with rotation error for pixels located on grain boundaries (as obtained from the EBSD file) being set to 0 . The error is of the order of $10^{-3}$ to $10^{-2}$ degree, with an observable concentration of measurement error. In the calculation with 3\% macroscopic strain, the average rotation of intra-grain pixels is 0.75 degree and the average rotation error is $2.6 \times 10^{-3}$ degree. As a result, the present procedure can measure disorientations due to plastic deformation extremely precisely, with an error less than $0.5 \%$.

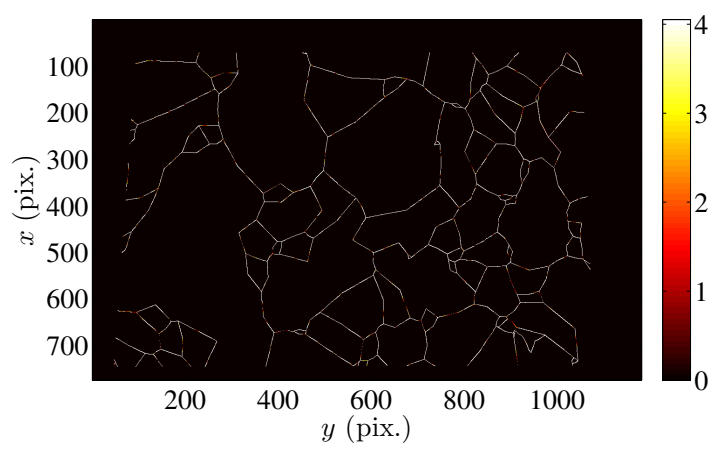

(a) $\Delta \theta$

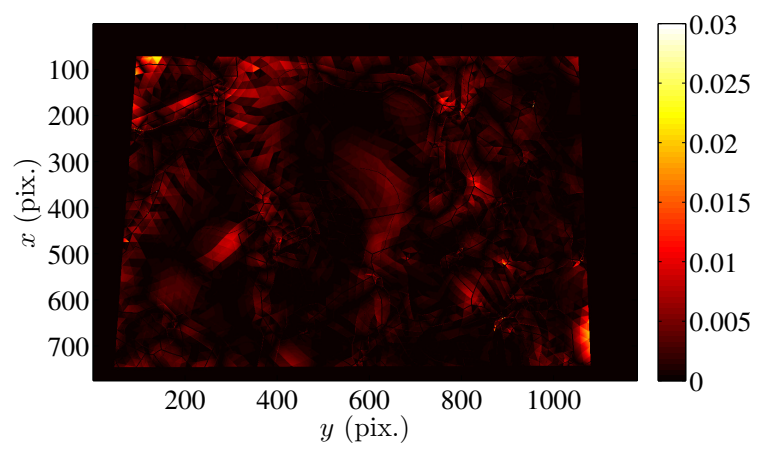

(b) Intra-grain $\Delta \theta$

Figure 9: (a) Rotation difference $\Delta \theta$ (degrees) between quaternion correlation results and $\mathrm{FE}$ computed values. (b) $\Delta \theta$ after canceling out the grain boundary values, thus intra-grain values become visible

It should be noted that the isotropic elastic regularization does not correspond to the simulation based on crystal plasticity. However, as the previous results have shown, isotropic elastic regularization gives a fair interpolation. This result is very convenient for the registration of experimental EBSD pictures. It is concluded that the displacements measured by the correlation procedure proposed herein are very close to those calculated by FE simulation. Besides, the residual field corresponds to the simulated rotation field except along grain boundaries. The measurement error is quite low (i.e., 0.3 pixel for the displacement and about $3 \times 10^{-3}$ degree for crystal rotation) although image contrast essentially exists at grain boundaries. Therefore, the algorithm is deemed validated.

\section{Application to experimental maps}

Inconel 600 , which is a nickel-based alloy, is used to carry out an in-situ tensile experiment. A $10 \times 2 \times 1 \mathrm{~mm}^{3}$ tensile sample is machined. Mechanical polishing of the sample surface is performed with cloths and diamond suspension down to $0.25 \mu \mathrm{m}$ followed by a finishing with $40-\mathrm{nm}$ colloidal silica suspension for 45 minutes. The sample is subjected to an in-situ tensile test at a strain rate of $4 \times 10^{-4} \mathrm{~s}^{-1}$ up to $20 \%$ longitudinal strain.

The SEM used in this study is a TESCAN Mira3 600. Backscattered electron detector in Z-contrast has been used, with an acceleration voltage of $9 \mathrm{kV}$ and a working distance of $17 \mathrm{~mm}$. The physical size of one pixel is $250 \mathrm{~nm}$. This resolution is chosen to ensure that a sufficient number of grains could be observed and each of them with a significant number of pixels. The scanned area is $500 \times 500 \mu \mathrm{m}^{2}$ with a definition of $2000 \times 2000$ pixels. The data obtained by EBSD acquisitions, and processed with OIM $^{\mathrm{TM}}$ software are used as a starting point of the analysis. The . ang file contains the measurement coordinates, three Euler angles, image quality index and confidence index of each pixel.

A series of EBSD analyses performed in the course of an in-situ tensile experiment on the specimen has been used to test the quaternion-based registration algorithm. Figure 10 shows two inverse pole figures in the reference and deformed states as obtained from $\mathrm{OIM}^{\mathrm{TM}}$. Figure $10(\mathrm{~b})$ corresponds to the raw data used to extract the quaternion fields shown in Figures 1 and 2.

From these maps, a region of interest is selected (Figure 11(a)) in which variable grain sizes are observed. Figure 11(b) shows the disorientation between the reference and deformed images (as obtained by a raw discrepancy $1-\Re\left[\boldsymbol{f g}^{-1}\right]$ ), which exhibits a "ribbon" shape where the ribbon width reveals the displacement magnitude and orientation, the color indicates the disorientation value. The mesh used for quaternion correlation consists of triangular elements with a rather uniform size (here about 30 pixels) spanning the chosen ROI but not conforming with the grain shapes (Figure 11(c)). 


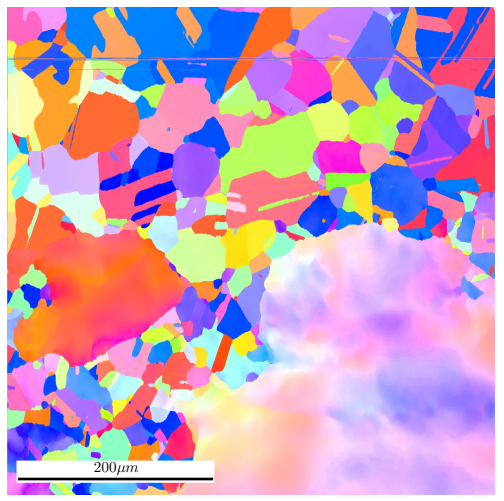

(a) Reference image

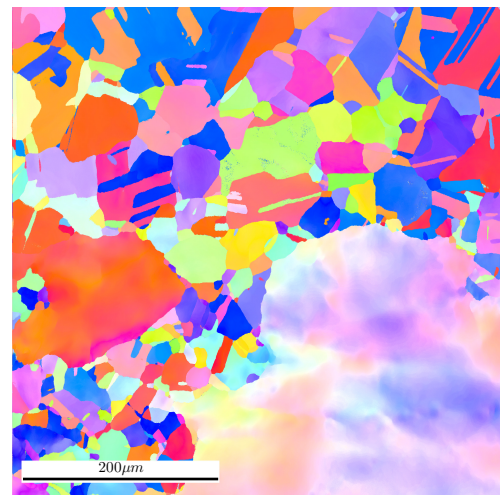

(b) Deformed image

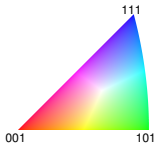

Figure 10: Inverse pole figures of the reference (a) and deformed (b) configurations

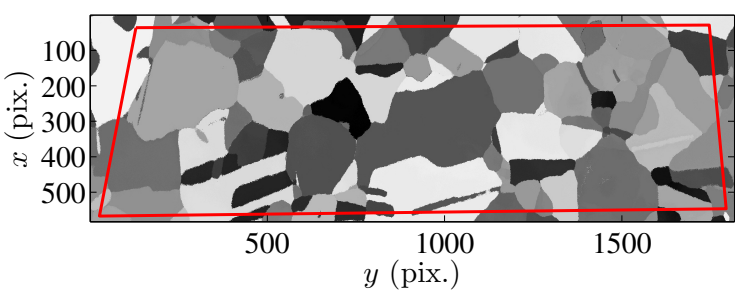

(a)

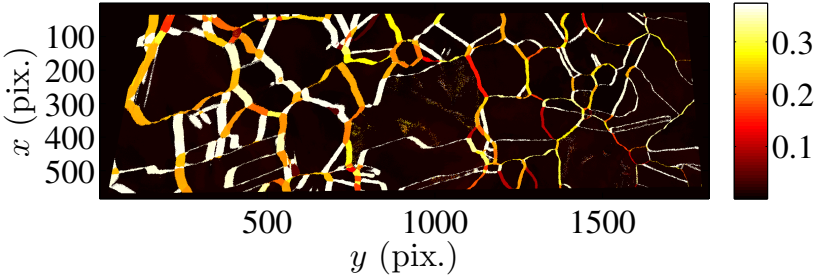

(b)

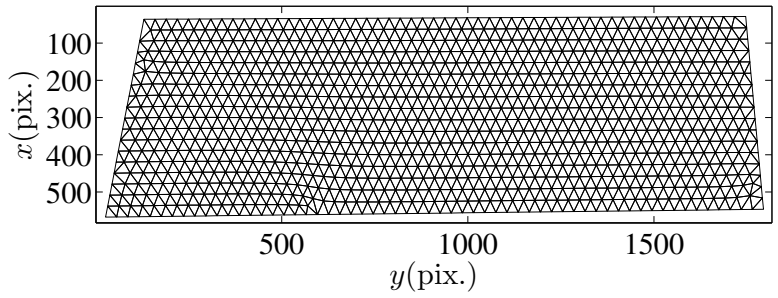

(c)

Figure 11: (a) ROI of the reference EBSD image. (b) Initial residual between the reference and deformed images. (c) FE mesh of the quaternion correlation calculation

The convergence criterion is still set to $5 \times 10^{-5}$ pixel, which is a very low value given the fact that only grain boundaries are highly contrasted. The elastic regularization length $\ell_{m}$ is initially chosen to be 400 pixels, and decreased to 200 pixels once convergence is obtained. The value of $\xi$ decreases from 16 to 1 pixel, and is followed by no filtering at all (i.e., $\xi=0$ ). The final value of $\ell_{m}$ is greater than the 100-pixel level adopted in the previous case study because the largest grain size is somewhat larger. A total of 800 iterations is needed to reach convergence. With the same implementation as for Section 4, each iteration requires about $30 \mathrm{~s}$.

The measured displacement field in $x$ and $y$ directions is shown in Figure 12(a-b). Figure 12(c) displays the corresponding crystal rotation field between the two image acquisitions. It is observed that in this experimental case the rotations are specific to grains, with discontinuities across grain boundaries just as in the FE simulation (Figure 8(a)).

The residual of quaternion correlation is shown in Figure 13(a). Grain boundaries are still visible, though very thin. Isolated intra-grain points can also be noticed. They are due to EBSD acquisition noise. Most of the grain boundaries are not wider than 3 pixels (see Figure 13(b)) and the emergence of several bright zones indicates substantial crystal rotations during tension, which is presumably due to crystal twinning. Figures 13(c) and 13(d) show the confidence index of the reference image and the displacement-corrected deformed image of the corresponding region. It is concluded that at triple points the confidence index is lower than for regular grain boundaries, i.e., the crystal orientation is not well determined and leads to high residuals. The quaternion correlation procedure cannot produce results that are more reliable than the exploited starting EBSD information. 


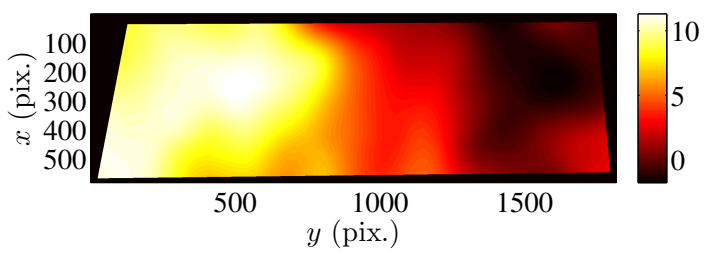

(a) $u_{x}$ (pixels)

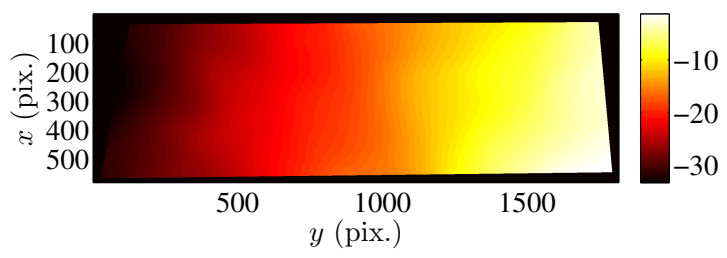

(b) $u_{y}$ (pixels)

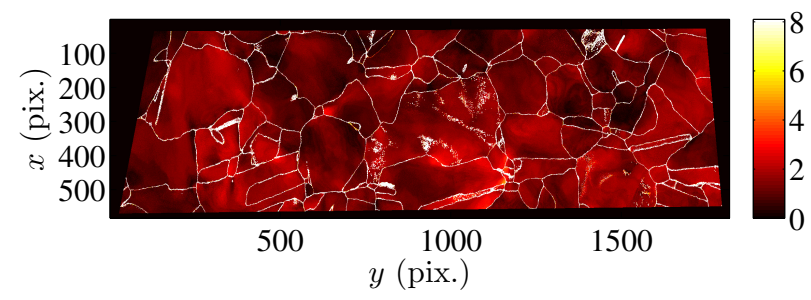

(c) $\theta$ (degrees)

Figure 12: Quaternion correlation results. (a-b) Measured displacement field in $x$ and $y$ directions (in pixels). (c) Corresponding rotation angle $\theta$ field (in degrees)

In order to validate the result, it is natural to consider the final residual, i.e., disorientation angle after registration. However, as discussed above, within grains this quantity is not exactly an error, namely, it corresponds to the actual lattice rotation that takes place in the plastic flow. The other part of the residual is the one that is supported by the grain boundaries. It is the latter that validates the convergence of the procedure. As shown in the magnified region (Figure 13(b)) the final residuals are essentially localized on grain boundaries. It is noteworthy that they are even more concentrated than what the confidence index would suggest.

\section{Conclusion and perspectives}

The algorithm introduced herein evaluates displacements based on crystallographic orientations re-encoded as quaternions instead of gray levels. Its main application deals with EBSD acquisitions. Displacement fields are measured and correlation residuals quantify the crystallographic rotation between images. Two cases have been studied. First, a synthetic case for which the displacement and rotation fields were known thanks to a crystal plasticity computation. The algorithm was deemed validated and the error levels of the algorithm were quantified. Second, EBSD maps acquired during an in-situ tensile test on a nickel-based alloy show that such a technique can also be applied to experimental cases. Consequently, acquisition noise does not hinder such technique. However, drift and other artifacts related to EBSD imaging need to be quantified.

The global lack of image contrast, except at grain boundaries, makes the correlation very challenging. Consequently, convergence is slow in comparison with registrations of speckle patterns. Pixel-wise crystal symmetry application at each step leads to additional computation steps. Standard DIC results based on the EBSD quality map treated as speckles can be used to initialize quaternion correlation, which speeds-up the calculation in practice. Besides, the discontinuity of crystal orientations at grain boundaries makes the convergence very difficult near the minimum point.

The measurement of kinematic and rotation fields at microstructure scale opens many possibilities in materials science. For example, the quaternion correlation algorithm can be used in the detection of crack tip, as the propagation of crack induces crystallographic rotations. Both displacement and rotation fields can be used for the study of plasticity and for the identification of constitutive laws. The algorithm can also be adapted to filter a series of consecutive EBSD results. As shown in Figure 13(a), isolated intra-grain high residuals indicate the noise of EBSD acquisitions either in the reference or deformed images. The algorithm provides a way of locating these spurious pixels and filter them out. Apart from detecting 'bad' pixels inside grains, the algorithm can also be used to adjust grain boundaries. Due to the discrete sampling effect, EBSD gives more rigged grain boundaries than the actual ones. Correspondence of pixels at grain boundaries has an 'averaging' effect, which helps to straighten grain boundaries. This mechanism can also be understood as several EBSD acquisitions actually improve the spatial resolution on the grain boundary, under the assumption that the grain boundary does not change much between 


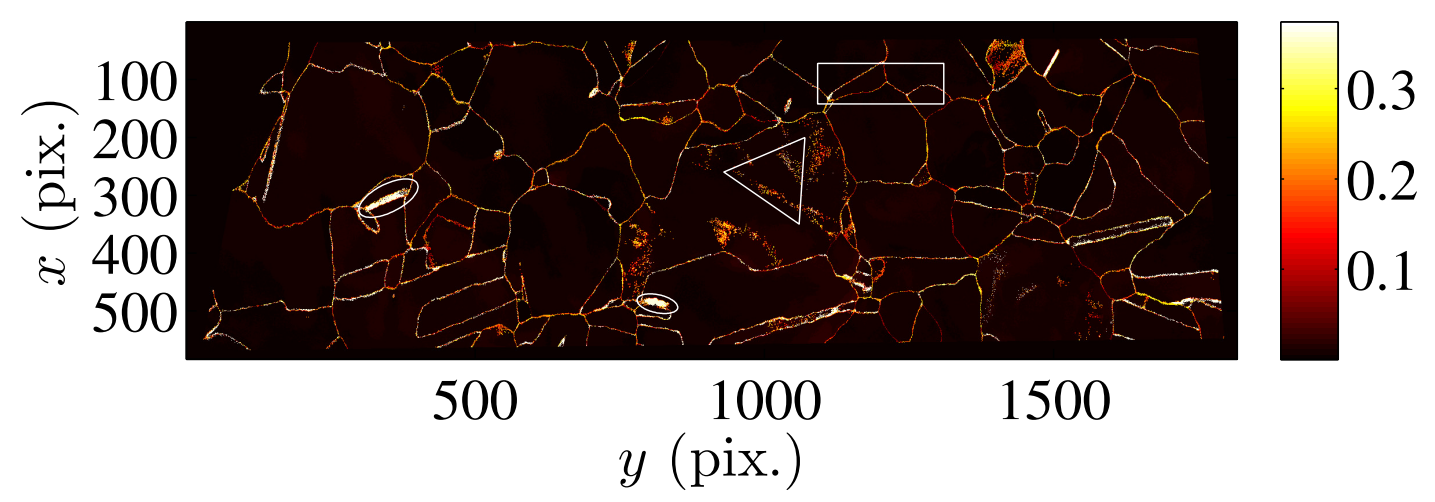

(a)

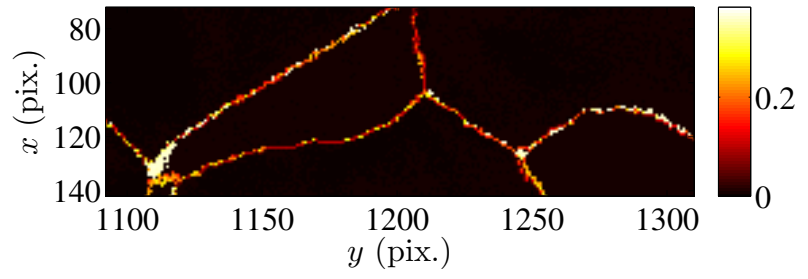

(b)

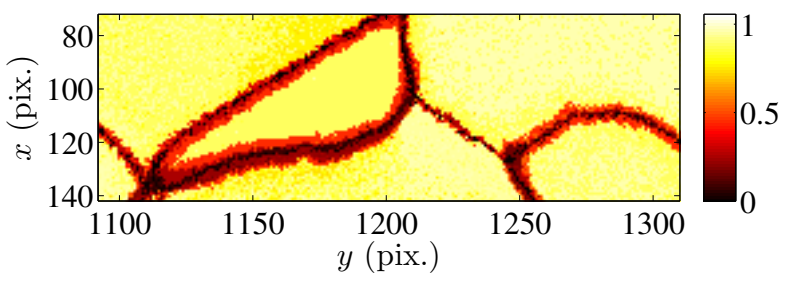

(c)

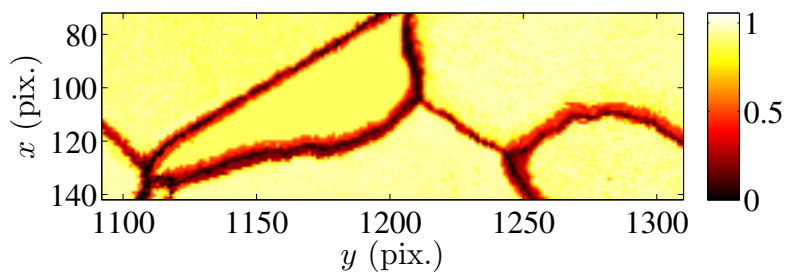

(d)

Figure 13: (a) Residual map in analyzed ROI. The isolated intra-grain high residuals in the triangle indicate EBSD acquisition noise. The bright zones shown in the ellipses indicate substantial crystal rotations during tension, presumably crystal twinning. The rectangle indicates the enlarged area shown in (b-d). (b) Zoomed residual map. Confidence index in the corresponding area in reference and deformed images are shown respectively in (c) and (d)

acquisitions.

\section{Acknowledgments}

The authors acknowledge the financial support of EDF with R\&D MAP and PERFORM projects. Nicolas Brynaert, Domonique Loisnard, Julien Stodolna and the electronic microscopy laboratory at Les Renardières are thanked for their help in the SEM acquisitions. They also thank Dr. Adrien Guery for constructive advices. 


\section{Appendix A: Basic properties of quaternions}

Multiplication rules for elementary quaternions reads

$$
\begin{aligned}
& \boldsymbol{i} \times \boldsymbol{i}=-1, \quad \boldsymbol{j} \times \boldsymbol{j}=-1, \quad \boldsymbol{k} \times \boldsymbol{k}=-1 \\
& \boldsymbol{i} \times \boldsymbol{j}=\boldsymbol{k}, \quad \boldsymbol{j} \times \boldsymbol{k}=\boldsymbol{i}, \quad \boldsymbol{k} \times \boldsymbol{i}=\boldsymbol{j} \\
& j \times i=-k, \quad k \times j=-i, \quad i \times k=-j
\end{aligned}
$$

The multiplicative sign $\times$ was shown explicitly in order to avoid possible confusions. In particular, the third line shows that the product is not commutative, and thus special care is to be exercised. In the following, to simplify notations, the product sign will be omitted and $\boldsymbol{q}_{1} \times \boldsymbol{q}_{2}$ will be denoted $\boldsymbol{q}_{1} \boldsymbol{q}_{2}$. The conjugate of a quaternion $\boldsymbol{q}=a+b \boldsymbol{i}+c \boldsymbol{j}+d \boldsymbol{k}$ is defined as

$$
\boldsymbol{q}^{*}=a-b \boldsymbol{i}-c \boldsymbol{j}-d \boldsymbol{k}
$$

and its quadratic norm is

$$
\|\boldsymbol{q}\|^{2}=\boldsymbol{q} \boldsymbol{q}^{*}=a^{2}+b^{2}+c^{2}+d^{2}
$$

A unit quaternion $\boldsymbol{U}_{q}$ is such that its norm is unity, and hence its inverse is its conjugate

$$
\boldsymbol{U}_{q}^{-1}=\boldsymbol{U}_{q}^{*}
$$

The multiplication of two unit quaternions produces a new unit quaternion, its corresponding rotation is the combination of the two original quaternions. The 'division' of two unit quaternions $\boldsymbol{p}$ and $\boldsymbol{q}, \boldsymbol{q} \boldsymbol{p}^{-1}$, indicates the rotation between them, with the following relationship between rotation angle $\theta$ and $\left(\boldsymbol{q p}^{-1}\right)_{1}$

$$
\left(\boldsymbol{q p}^{-1}\right)_{1}=\cos \frac{\theta}{2} \approx 1-\frac{\theta^{2}}{8}, \text { with small } \theta
$$

Linear quaternion interpolation (Lerp) reads

$$
L(\boldsymbol{p}, \boldsymbol{q}, h)=\boldsymbol{p}(1-h)+\boldsymbol{q} h
$$

Linear quaternion interpolation needs to be projected onto the unit sphere in 4D to obtain unitary quaternions

$$
U_{L(\boldsymbol{p}, \boldsymbol{q}, h)}=\frac{\boldsymbol{p}(1-h)+\boldsymbol{q} h}{\|\boldsymbol{p}(1-h)+\boldsymbol{q} h\|}
$$

Figure 14 illustrates the Lerp of quaternions in 2 dimension. The secant pq is equally split by Lerp, while the angle between $\boldsymbol{p}$ and $\boldsymbol{q}$ is not. Lerp is easy to implement, namely, component-wise linear interpolation and renormalization.

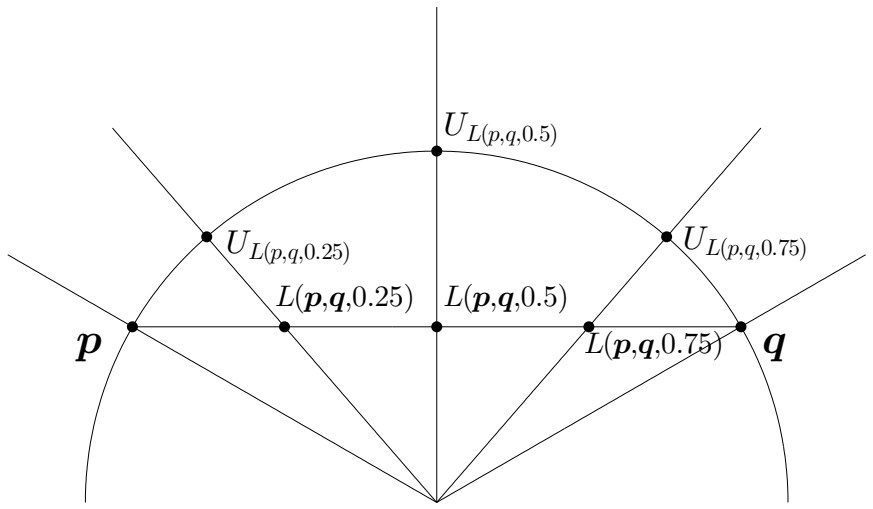

Figure 14: Lerp illustrated in the orientation space. Quaternions $\boldsymbol{p}$ and $\boldsymbol{q}$ are shown in orientation space with their linear interpolations

All representation of rotations, namely, Euler angle notation, axis-angle notation and quaternion are interchangeable. Their transformation can be performed by comparison of the rotation matrices. The rotation matrix based on Euler angles $\left(\varphi_{1}, \Phi, \varphi_{2}\right)$ reads in Bunge notations

$$
\left[\begin{array}{ccc}
\cos \varphi_{1} \cos \varphi_{2}-\sin \varphi_{1} \sin \varphi_{2} \cos \Phi & \sin \varphi_{1} \cos \varphi_{2}+\cos \varphi_{1} \sin \varphi_{2} \cos \Phi & \sin \varphi_{2} \sin \Phi \\
-\cos \varphi_{1} \sin \varphi_{2}-\sin \varphi_{1} \cos \varphi_{2} \cos \Phi & -\sin \varphi_{1} \sin \varphi_{2}+\cos \varphi_{1} \cos \varphi_{2} \cos \Phi & \cos \varphi_{2} \sin \Phi \\
\sin \varphi_{1} \sin \Phi & -\cos \varphi_{1} \sin \Phi & \cos \Phi
\end{array}\right]
$$


The rotation matrix based on axis-angle $\left(\theta,\left[d_{1}, d_{2}, d_{3}\right]\right)$ is written as

$$
\left[\begin{array}{ccc}
\left(1-d_{1}^{2}\right) \cos \theta+d_{1}^{2} & d_{1} d_{2}(1-\cos \theta)+d_{3} \sin \theta & d_{1} d_{3}(1-\cos \theta)-d_{2} \sin \theta \\
d_{1} d_{2}(1-\cos \theta)-d_{3} \sin \theta & \left(1-d_{2}^{2}\right) \cos \theta+d_{2}^{2} & d_{2} d_{3}(1-\cos \theta)+d_{1} \sin \theta \\
d_{1} d_{3}(1-\cos \theta)+d_{2} \sin \theta & d_{2} d_{3}(1-\cos \theta)-d_{1} \sin \theta & \left(1-d_{3}^{2}\right) \cos \theta+d_{3}^{2}
\end{array}\right]
$$

The quaternion $\left(q_{1}, q_{2}, q_{3}, q_{4}\right)$ and axis-angle notation $\left(\theta,\left[d_{1}, d_{2}, d_{3}\right]\right)$ are related as

$$
q_{1}=\cos \frac{\theta}{2}, q_{2}=\sin \frac{\theta}{2} d_{1}, q_{3}=\sin \frac{\theta}{2} d_{2}, q_{4}=\sin \frac{\theta}{2} d_{3}
$$




\section{Appendix B: 48 unitary quaternions corresponding to cubic crystal sym- metry}

There are 24 crystal symmetry systems in a cubic crystal:

- 3-fold rotation symmetry in cubic diagonal direction $\{111\}$

- 4-fold rotation symmetry in orthogonal direction $\{100\}$

- 2-fold rotation symmetry in facial diagonal direction $\{110\}$

Crystal symmetry can be represented by unit quaternion. For example, a rotation of $\theta=2 \pi / 3$ about $\boldsymbol{d}=$ $(1 / \sqrt{3})(1,1,1)$ can be represented as

$$
\boldsymbol{p}=\left(\cos (\pi / 3), d_{1} \sin (\pi / 3), d_{2} \sin (\pi / 3), d_{3} \sin (\pi / 3)\right)=(1 / 2)(1,1,1,1)
$$

All of the 48 cubic symmetry quaternions $\boldsymbol{p}_{i}^{\text {cub }}$ are listed in Table 3.

Table 3: List of the 48 quaternions image of $\boldsymbol{q}_{0}=1$ in a cubic symmetry. For the sake of simplicity a constant scale factor is factorized in the first column

\begin{tabular}{rrrrr||rrrrr} 
Scale factor & $p_{1}$ & $p_{2}$ & $p_{3}$ & $p_{4}$ & Scale factor & $p_{1}$ & $p_{2}$ & $p_{3}$ & $p_{4}$ \\
\hline 1 & +1 & 0 & 0 & 0 & 1 & -1 & 0 & 0 & 0 \\
1 & 0 & +1 & 0 & 0 & 1 & 0 & -1 & 0 & 0 \\
1 & 0 & 0 & +1 & 0 & 1 & 0 & 0 & -1 & 0 \\
1 & 0 & 0 & 0 & +1 & 1 & 0 & 0 & 0 & -1 \\
$1 / 2$ & +1 & +1 & +1 & +1 & $1 / 2$ & -1 & -1 & -1 & -1 \\
$1 / 2$ & +1 & -1 & -1 & -1 & $1 / 2$ & -1 & +1 & +1 & +1 \\
$1 / 2$ & +1 & +1 & -1 & +1 & $1 / 2$ & -1 & -1 & +1 & -1 \\
$1 / 2$ & +1 & -1 & +1 & -1 & $1 / 2$ & -1 & +1 & -1 & +1 \\
$1 / 2$ & +1 & -1 & +1 & +1 & $1 / 2$ & -1 & +1 & -1 & -1 \\
$1 / 2$ & +1 & +1 & -1 & -1 & $1 / 2$ & -1 & -1 & +1 & +1 \\
$1 / 2$ & +1 & -1 & -1 & +1 & $1 / 2$ & -1 & +1 & +1 & -1 \\
$1 / 2$ & +1 & +1 & +1 & -1 & $1 / 2$ & -1 & -1 & -1 & +1 \\
$\sqrt{2} / 2$ & +1 & +1 & 0 & 0 & $\sqrt{2} / 2$ & -1 & -1 & 0 & 0 \\
$\sqrt{2} / 2$ & +1 & 0 & +1 & 0 & $\sqrt{2} / 2$ & -1 & 0 & -1 & 0 \\
$\sqrt{2} / 2$ & +1 & 0 & 0 & +1 & $\sqrt{2} / 2$ & -1 & 0 & 0 & -1 \\
$\sqrt{2} / 2$ & +1 & -1 & 0 & 0 & $\sqrt{2} / 2$ & -1 & +1 & 0 & 0 \\
$\sqrt{2} / 2$ & +1 & 0 & -1 & 0 & $\sqrt{2} / 2$ & -1 & 0 & +1 & 0 \\
$\sqrt{2} / 2$ & +1 & 0 & 0 & -1 & $\sqrt{2} / 2$ & -1 & 0 & 0 & +1 \\
$\sqrt{2} / 2$ & 0 & +1 & +1 & 0 & $\sqrt{2} / 2$ & 0 & -1 & -1 & 0 \\
$\sqrt{2} / 2$ & 0 & -1 & +1 & 0 & $\sqrt{2} / 2$ & 0 & +1 & -1 & 0 \\
$\sqrt{2} / 2$ & 0 & 0 & +1 & +1 & $\sqrt{2} / 2$ & 0 & 0 & -1 & -1 \\
$\sqrt{2} / 2$ & 0 & 0 & -1 & +1 & $\sqrt{2} / 2$ & 0 & 0 & +1 & -1 \\
$\sqrt{2} / 2$ & 0 & +1 & 0 & +1 & $\sqrt{2} / 2$ & 0 & -1 & 0 & -1 \\
$\sqrt{2} / 2$ & 0 & -1 & 0 & +1 & $\sqrt{2} / 2$ & 0 & +1 & 0 & -1
\end{tabular}




\section{References}

[1] A. Allais, M. Bornert, T. Bretheau, and D. Caldemaison. Experimental characterization of the local strain field in an heterogeneous elastoplastic material. Acta Metalurgica Materialia, 42(11):3865-3880, 1994.

[2] F. Amiot, J. N. Périé, and S. Roux. Equilibrium Gap Method, pages 331-362. John Wiley \& Sons, Inc., 2012.

[3] F. Barbe, L. Decker, D. Jeulin, and G. Cailletaud. Intergranular and intragranular behavior of polycrystalline aggregates. Part 1: F.E. model. International Journal of Plasticity, 17(4):513-536, 2001.

[4] M. Bertin, C. Du, J.P.M. Hoefnagels, and F. Hild. Crystal plasticity parameter identification with 3d measurements and integrated digital image correlation. Acta Materialia (in press), 2016.

[5] M. Berveiller and A. Zaoui. An extension of the self-consistent scheme to plastically-flowing polycrystals. Journal of The Mechanics and Physics of Solids, 26:325-344, 1979.

[6] J. Carroll, C. Efstathiou, J. Lambros, H. Sehitoglu, B. Hauber, S. Spottswood, and R. Chona. Investigation of fatigue crack closure using multiscale image correlation experiments. Engineering Fracture Mechanics, $76(15): 2384-2398,2009$.

[7] J.H. Cho, A.D. Rollett, and K.H. Oh. Determination of a mean orientation in electron backscatter diffraction measurements. Metallurgical and Materials Transactions A, 36(12):3427-3438, 2005.

[8] Code_Aster. www.code-aster.org. 2015.

[9] N.M. Cordero, S. Forest, E.P. Busso, S. Berbenni, and M. Cherkaoui. Grain size effects on plastic strain and dislocation density tensor fields in metal polycrystals. Computational Materials Science, 52(1):7-13, 2012.

[10] P. Doumalin and M. Bornert. Micromechanical applications of digital image correlation techniques. In Pierre Jacquot and Jean-Marc Fournier, editors, Interferometry in Speckle Light, pages 67-74. Springer Berlin Heidelberg, 2000.

[11] A. Guery, F. Hild, F. Latourte, and S. Roux. Identification of crystal plasticity parameters using dic measurements and weighted femu. Mechanics of Materials, 100:55 - 71, 2016.

[12] A. Guery, F. Hild, F. Latourte, and S. Roux. Slip activities in polycrystals determined by coupling DIC measurements with crystal plasticity calculations. International Journal of Plasticity, 81:249 - 266, 2016.

[13] A. Guery, F. Latourte, F. Hild, and S. Roux. Characterization of SEM speckle pattern marking and imaging distortion by digital image correlation. Measurement Science and Technology, 25:12pp, 2014.

[14] M.E. Gurtin, L. Anand, and S.P. Lele. Gradient single-crystal plasticity with free energy dependent on dislocation densities. Journal of the Mechanics and Physics of Solids, 55(9):1853 - 1878, 2007.

[15] W.R. Hamilton. Lectures on Quaternions. Dublin : Hodges and Smith, 1853.

[16] W.R. Hamilton. Elements of Quaternions. London, Longmans, Green, \& co, 1866.

[17] J.H. Han, K.K. Jee, and K.H. Oh. Orientation rotation behavior during in situ tensile deformation of polycrystalline 1050 aluminum alloy. International Journal of Mechanical Sciences, 45(10):1613 - 1623, 2003. 6th Asia-Pacific Symposium on Advances in Engineering Plasticity and its Applications.

[18] F. Hild and S. Roux. Digital Image Correlation. Wiley-VCH, 2012.

[19] T. Hoc, J. Crépin, L. Gélébart, and A. Zaoui. A procedure for identifying the plastic behavior of single crystals from the local response of polycrystals. Acta Materialia, 51(18):5477-5488, 2003.

[20] M.F. Horstemeyer. Integrated Computational Materials Engineering (ICME) for Metals: Using Multiscale Modeling to Invigorate Engineering Design with Science. Wiley-TMS, 2012.

[21] E. Héripré, M. Dexet, J. Crépin, L. Gélébart, A. Roos, M. Bornert, and D. Caldemaison. Coupling between experimental measurements and polycrystal finite element calculations for micromechanical study of metallic materials. International Journal of Plasticity, 23(9):1512-1539, 2007. 
[22] H. Jin, W.Y. Lu, S. Haldar, and H.A. Bruck. Microscale characterization of granular deformation near a crack tip. Journal of Materials Science, 46(20):6596-6602, 2011.

[23] H.D. Joo, J.S. Kim, K.H. Kim, N. Tamura, and Y.M. Koo. In situ synchrotron x-ray microdiffraction study of deformation behavior in polycrystalline coppers during uniaxial deformations. Scripta Materialia, 51(12):1183 - 1186, 2004.

[24] F. Latourte, T. Salez, A. Guery, N. Rupin, and M. Mahé. Deformation studies from in-situ SEM experiments of a reactor pressure vessel steel at room and low temperatures. Journal of Nuclear Materials, 454(1-3):373-380, 2014 .

[25] S. Leclercq, D. Lidbury, S.V. Dyck, D. Moinereau, A. Alamo, and A.A. Mazouzi. PERFORM 60 - prediction of the effects of radiation for reactor pressure vessel and in-core materials using multi-scale modelling - 60 years foreseen plant lifetime. Journal of Nuclear Materials, 406(1):193-203, 2010.

[26] H. Lim, J.D. Carroll, C.C. Battaile, B.L. Boyce, and C.R. Weinberger. Quantitative comparison between experimental measurements and CP-FEM predictions of plastic deformation in a tantalum oligocrystal. International Journal of Mechanical Sciences, 92(0):98 - 108, 2015.

[27] B.D. Lucas and T. Kanade. An iterative registration technique with an application to stereo vision. pages 121-130, 1981.

[28] A. Ma, F. Roters, and D. Raabe. On the consideration of interactions between dislocations and grain boundaries in crystal plasticity finite element modeling - Theory, experiments, and simulations. Acta Materialia, 54(8):2181-2194, 2006.

[29] G. Monnet, L. Vincent, and B. Devincre. Dislocation-dynamics based crystal plasticity law for the low- and high-temperature deformation regimes of bcc crystal. Acta Materialia, 61(16):6178-6190, 2013.

[30] C. Ru, Y. Zhang, Y. Sun, Y. Zhong, X. Sun, D. Hoyle, and I. Cotton. Automated four-point probe measurement of nanowires inside a scanning electron microscope. IEEE Transactions on Nanotechnology, 10:674-681, 2011.

[31] J. Réthoré, S. Roux, and F. Hild. An extended and integrated digital image correlation technique applied to the analysis of fractured samples. European Journal of Computational Mechanics, 18:285-306, 2009.

[32] M.A. Sutton. Computer vision-based, noncontacting deformation measurements in mechanics: a generational transformation. Applied Mechanics Reviews, 65(5):050802, 2013.

[33] M.A. Sutton, N. Li, D. Garcia, N. Cornille, J.J. Orteu, S.R. McNeill, H.W. Schreier, and X. Li. Metrology in a scanning electron microscope: theoretical developments and experimental validation. Measurement Science and Technology, 17:2613-2622, 2006.

[34] M.A. Sutton, N. Li, D.C. Joy, A.P. Reynolds, and X. Li. Scanning electron microscopy for quantitative small and large deformation measurements Part I: SEM imaging at magnifications from 200 to 10.000. Experimental Mechanics, 47(6):775-787, 2007.

[35] T. Taillandier-Thomas, S. Roux, T. F. Morgeneyer, and F. Hild. Localized strain field measurement on laminography data with mechanical regularization. Nuclear Instruments and Methods in Physics Research Section B: Beam Interactions with Materials and Atoms, 324(0):70-79, 2014.

[36] A. Tatschl and O. Kolednik. On the experimental characterization of crystal plasticity in polycrystals. Materials Science and Engineering: A, 356(1-2):447-463, 2003.

[37] A.N. Tikhonov and V.Y. Arsenin. Solution of ill-posed problems. Winston, New York (USA), 1977.

[38] Z. Tomičević, F. Hild, and S. Roux. Mechanics-aided digital image correlation. Journal of Strain Analysis for Engineering Design, 48:330-343, 2013.

[39] A.J. Wilkinson, G. Meaden, and D.J. Dingley. High-resolution elastic strain measurement from electron backscatter diffraction patterns: New levels of sensitivity. Ultramicroscopy, 106(4-5):307 - 313, 2006. 